\title{
New experimental classification of divertor detachment in ASDEX Upgrade
}

\author{
S. Potzel, M. Wischmeier, M. Bernert, R. Dux, H.W. Müller, \\ A. Scarabosio and ASDEX Upgrade Team \\ Max-Planck-Institut für Plasmaphysik, EURATOM Association, Boltzmannstraße 2, \\ 85748 Garching, Germany \\ E-mail: Steffen.Potzel@ipp.mpg.de
}

\begin{abstract}
In this article a new experimental classification of divertor detachment in ASDEX Upgrade is presented. For this purpose a series of Ohmic and L-mode density ramp discharges at different heating powers, magnetic field directions and plasma species were carried out. For the first time at ASDEX Upgrade the electron density in the divertor volume and the occurrence of volume recombination were measured by means of spectroscopy. It is shown that detachment is not a continuously evolving process but rather undergoes three distinct states while the characteristics of the inner and outer divertor are strongly coupled. Before the complete detachment of the inner and outer divertor, radiative fluctuations occur in the inner divertor close to the Xpoint, observed for the first time via new fast diode bolometers. Finally, the effect of an externally applied magnetic perturbation field on the detachment process is investigated.
\end{abstract}

\section{Introduction}

Avoiding damage of the divertor material by keeping the power load below a certain threshold is a major challenge of future fusion devices such as ITER. For tungsten, at least in the D-T campaign the foreseen ITER divertor target material, the power load must be kept below $P \approx 10 \mathrm{MW} / \mathrm{m}^{2}$ in continuous operation. This can in ITER only be achieved with the plasma being detached or partially detached $[1,2]$.

Divertor detachment is characterized by a strong reduction of the total particle flux to the target plates. With a reduction of the plasma temperature in front of the target volumetric processes become important $[3,4,5]$. At electron temperatures below $\approx 5 \mathrm{eV}$ recycled neutrals from the target undergo several charge exchange (CX) collisions with plasma ions before they are ionized. This results in a change from a free-streaming to a diffusive-like plasma flow towards the target, which leads to a broadening of the ion flux profile at the target. Although the total ion flux is not reduced by CX collisions, a reduction of the peak ion flux is achieved. Furthermore, at $T \lesssim 1.5 \mathrm{eV}$ plasma ions can recombine before reaching the target. This process leads to a strong reduction of the total ion flux and plasma pressure and to an increase of the neutral pressure in front 
of the target. The necessary temperature reduction can be achieved by increasing the plasma density via fueling or with an additional injection of impurities, which cool the divertor plasma via line radiation.

Plasma detachment has been experimentally investigated in many tokamaks and the qualitative mechanisms leading to detachment seem to be understood $[6,7,8]$. A theoretical description of the detachment process is based on strongly coupled nonlinear equations and a quantitative description is only possible through extensive code simulations. But even the most sophisticated codes are not able to reproduce all experimental observations related to divertor detachment of present day experiments [9], indicating that there might be some physical processes missing in the codes. In particular to be able to predict the ITER divertor performance a correct simulation of divertor detachment is indispensable.

For example, the onset of detachment, defined by the moment when the ion flux to the target starts to decrease as the plasma density is further increased (the so called roll over), happens in most tokamaks much earlier in the inner than in the outer divertor. Moreover, the inner divertor receives a higher ion flux and a lower power load before the onset of detachment compared to the outer divertor. Several reasons for these asymmetries have been discussed $[10,11,12,13,14]$. At present it is thought that they are primarily caused by drift flows $[15,16]$. Furthermore, the radial transport of plasma particles in the scrape-off layer (SOL) is unknown. In the codes it is usually assumed to be of diffusive nature. But it was shown that at medium to high densities intermittent turbulent transport can be dominant [17] and increases with density [18]. Hence, in a recent work a collisionality dependent diffusion coefficient $D_{\perp}$ was used instead of a constant one [19]. This yields better results with respect to the density where the roll over occurs.

A key to an improved description of detachment is a better knowledge of the plasma parameters in the divertor and the SOL. The aim of this paper is the detailed characterization of divertor detachment with new diagnostics available at ASDEX Upgrade. We present the spatio-temporal evolution of detachment in both, the inner and outer divertor. For this purpose a series of Ohmic and L-mode discharges were performed where detachment was achieved through density ramps. The experiments were carried out at different heating powers, magnetic field directions and plasma species. For the first time the electron density in the divertor volume and the occurrence of volume recombination were measured by means of spectroscopy. It is shown that detachment in ASDEX Upgrade is not a continuous process but rather undergoes three distinct states while the characteristics of the inner and outer divertor are strongly coupled.

The paper is organized as follows: in section 2 an overview of the experimental and diagnostic setup is given and methods determining the occurrence of volume recombination and the magnitude of detachment are introduced. The evolution of the detachment process is presented in detail in section 3. Section 4 discusses phenomena, which are observed here for the first time and a summary is given in section 5 . 


\section{Experiment and Diagnostic Setup}

\subsection{Discharge setup}

A series of ohmic and L-mode density ramp discharges has been performed at ASDEX Upgrade to study the detachment of the divertor. Since 2007, the plasma facing surfaces of ASDEX Upgrade are completely covered with tungsten [20]. All discharges of this series were in lower single null divertor configuration with a lower triangularity of $\delta=0.36$, a plasma current of $I_{p}=1 \mathrm{MA}$, a toroidal magnetic field of $B_{t}=2.5 \mathrm{~T}$ and a safety factor of $q_{95} \approx 4$. The electron cyclotron resonance heating, ECRH, power was varied from discharge to discharge. The fueling species was changed between deuterium and hydrogen and the field direction was changed between forward field (ion $\mathbf{B} \times \nabla \mathbf{B}$ drift towards the lower divertor) and reversed field, see Table 1. Due to the alignment of the divertor tiles, the magnetic helicity cannot be reversed. Thus $B_{t}$ and $I_{p}$ have to be changed simultaneously in reversed field operation. In addition, one discharge was performed with an externally applied magnetic perturbation field (MP), see Table 1.

Table 1. Table of plasma parameters that were varied during the series of density ramp discharges.

\begin{tabular}{ccccc}
\hline shot \# & $P_{E C R H}[\mathrm{~kW}]$ & Gas & Field & special \\
\hline 27098 & 0 & D & forward & \\
27099 & 400 & D & forward & \\
27100 & 600 & D & forward & \\
27101 & 900 & D & forward & \\
27102 & 900 & D & forward & MP field \\
27283 & 0 & D & reversed & \\
27284 & 900 & D & reversed & \\
27286 & 1300 & D & reversed & \\
27360 & 0 & H & forward & \\
27361 & 1000 & H & forward & \\
\hline
\end{tabular}

In Figure 1 time traces of various plasma parameters for the discharge \#27100 are shown. The current flat top was reached at $1.3 \mathrm{~s}$ and a stable magnetic configuration at $1.8 \mathrm{~s}$. The strike point positions where then kept constant within a tolerance of $1 \mathrm{~cm}$. This magnetic configuration in the divertor is shown in Figure 2, with the inner and outer scrape-off layer (SOL) or high and low field side divertor SOL, respectively. The strike point positions had to be kept constant to measure the temporal evolution of the divertor plasma excluding any geometric effect. The gas fueling ramp starts at $2 \mathrm{~s}$ and was increased from $1 \cdot 10^{21}$ up to $1 \cdot 10^{22}$ atoms $/ \mathrm{s}$ with a rate of $1 \cdot 10^{21} \mathrm{atoms} / \mathrm{s}^{2}$. Then the rate was increased to $3 \cdot 10^{21}$ atoms $/ \mathrm{s}^{2}$ and the fueling was ramped up until the density limit occurred. The fueling ramp leads to a continuous increase of the plasma density and to the development of the detachment in the divertor. The same fueling scheme was used for all discharges. 


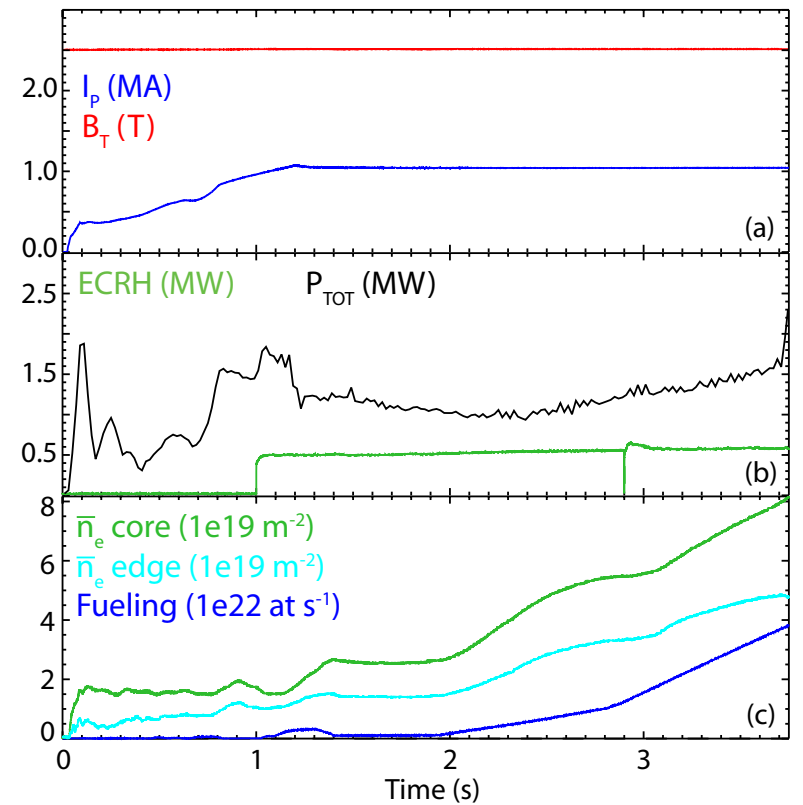

Figure 1. Time traces of a representative discharge \#27100. $I_{p}$ and $B_{t}$ (a), applied ECRH power and total heating power including ohmic heating (b), plasma fueling and central and edge line integrated plasma density (c)

\subsection{Diagnostic setup}

Flush mounted Langmuir triple probes (LP) measure the ion saturation current density, $j_{\text {sat }}$, and the electron density, $n_{e, t}$ and temperature, $T_{e, t}$ at the divertor targets at the positions shown in Figure $2 \mathrm{a}$. The ion flux, $\Gamma_{D^{+}}$, is then calculated dividing $j_{\text {sat }}$ by the elementary charge $e$, assuming pure deuterium plasmas. The spatial probe separation in poloidal direction is $\approx 2 \mathrm{~cm}$ in the inner and $\approx 2.5 \mathrm{~cm}$ in the outer divertor. The acquisition time is $\Delta t=0.035 \mathrm{~ms}$.

The electron density in the divertor volume, $n_{e, V}$, is determined via a spectroscopic measurement of the Stark broadened $\mathrm{D}_{\epsilon}$ line. This method is commonly used on other devices, such as JET, MAST or C-Mod [21, 22, 8]. Here, synthetic Stark broadened line shapes, calculated and published by Stehlé [23] based on the model microfield method, are convoluted with the instrument function and the Doppler broadening of a fixed neutral temperature of $T_{n}=5 \mathrm{eV}$. These shapes are fitted to the measured lines in a least square sense, in which $n_{e, V}$ is a fit parameter. For $n_{e, V} \geq 4 \cdot 10^{19} \mathrm{~m}^{-3}$ and $B_{t} \leq 3 \mathrm{~T}$ the Zeeman splitting and variations of $T_{n}$ can be neglected (for details see [24]). This measurement has an uncertainty of $15 \%$, an acquisition time of $\Delta t=2.65 \mathrm{~ms}$ and is a line integrated measurement weighted with the $\mathrm{D}_{\epsilon}$ emissivity along the lines of sight (LOS) which are shown in Figure 2. Together with the crossed LOS the measurement can approximately be localized by comparing it with the total radiation distribution measured by bolometry. If recombination processes can be neglected, the $\mathrm{D}_{\epsilon}$ emission profile is comparable to the distribution of the total radiation. Recombination preferably populates the higher $n$-levels. Thus, if recombination is dominant, the $\mathrm{D}_{\epsilon}$ emission is 
not comparable anymore to the total radiation, which is still dominated by ionization processes.

Fast AXUV diode bolometers measure the radiation between $1 \mathrm{eV}$ and $8 \mathrm{keV}$ [25]. This measurement is not absolutely calibrated and has a time resolution of $5 \mu \mathrm{s}$. The positions of the the bolometer chords are shown in Figure $2 \mathrm{~b}$.
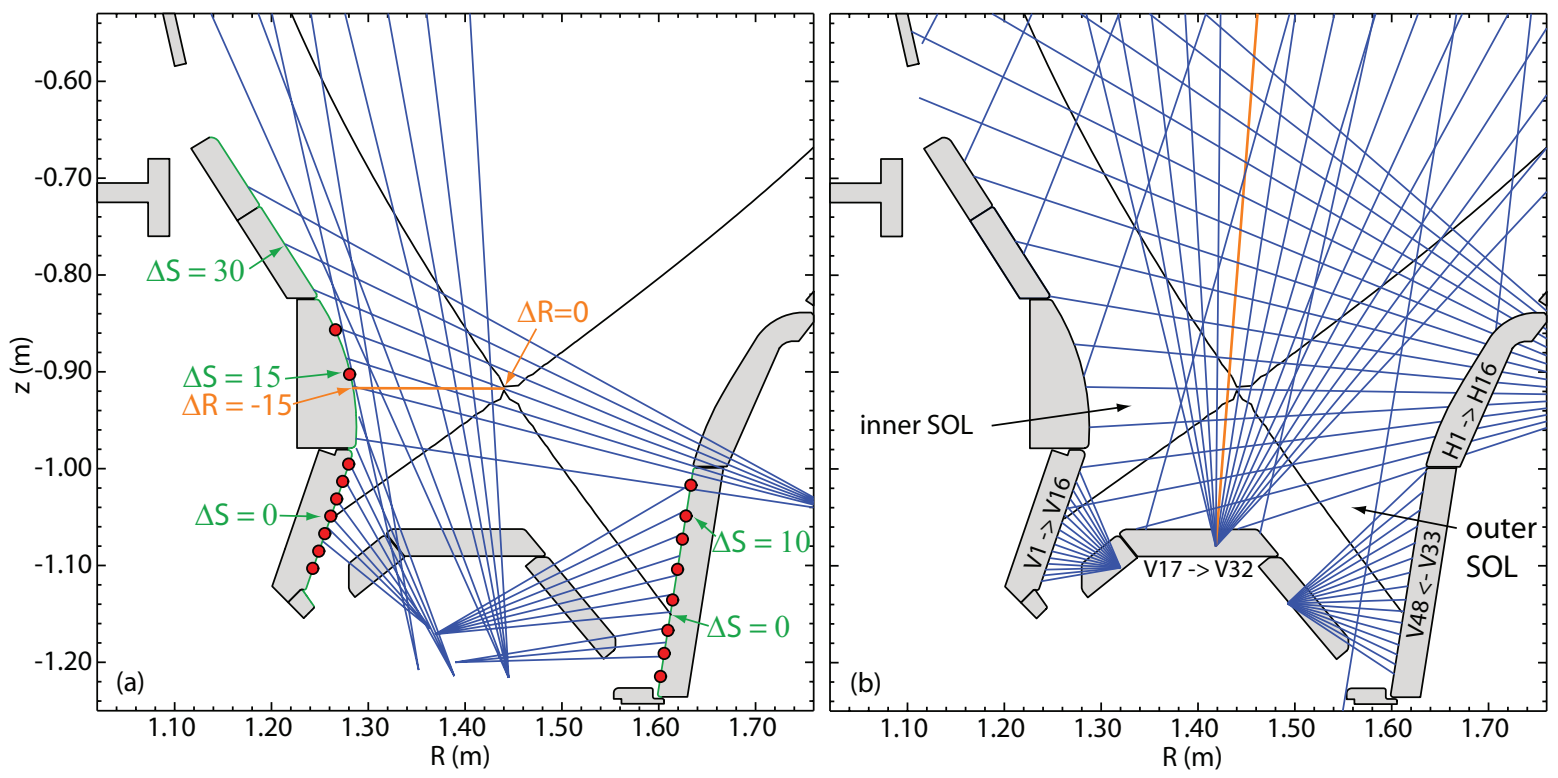

Figure 2. (a): LOS for Stark broadening (blue) and fixed Langmuir probes (red). Also shown are the $\Delta S$ and $\Delta R$ coordinates. (b): AXUV LOS (blue).

\subsection{Spectroscopic determination of volume recombination}

By measuring the line emission ratio of two different transitions of the same atom it is in principle possible to determine the electron temperature. Therefore it is necessary that the line emissions of both transitions weakly depend on the electron density. For example, this is routinely done at TEXTOR by measuring helium line ratios [26] or at JT-60U and JET by measuring Balmer line ratios [27, 28]. With the spectrometers used for this work, it is possible to measure the Balmer $\mathrm{D}_{\delta}$ and $\mathrm{D}_{\epsilon}$ lines simultaneously. As these are line integrated measurements, our aim was not to deduce absolute temperatures out of the $\mathrm{D}_{\delta} / \mathrm{D}_{\epsilon}$ ratio, but to deduce whether the divertor plasma is dominated by ionization or recombination.

The emissivity of the Balmer $D_{\delta}$ line, $n=6 \rightarrow n=2$, is written as:

$$
\begin{aligned}
\epsilon_{6 \rightarrow 2} & =n_{e} n_{D}^{+0} \operatorname{PEC}^{e x c}\left(n_{e} T_{e}\right)+n_{e} n_{D}^{+1} \operatorname{PEC}^{r e c}\left(n_{e} T_{e}\right) \\
& =n_{e} n_{D} \underbrace{\left(f^{+0} \operatorname{PEC}^{e x c}\left(n_{e} T_{e}\right)+f^{+1} \operatorname{PEC}^{r e c}\left(n_{e} T_{e}\right)\right)}_{\text {TEC }}
\end{aligned}
$$

with $n_{D}^{+0}$ and $n_{D}^{+1}$ the neutral deuterium and ionized deuterium densities, respectively. The PECs are the so-called Photon Emissivity Coefficients for recombination and 
excitation and have been calculated for various elements and transitions by the ADAS project $\ddagger$ based on a Collisional Radiative Model [29]. The $\mathrm{PEC}^{e x c}$ and $\mathrm{PEC}^{\text {rec }}$ are illustrated in Figure 3a for two different densities. It can be seen that the recombination process dominates with respect to ionization when $T<1.5 \mathrm{eV}$. The fractional abundance, $f$, in equation 1 is the ratio of the neutral or ionized deuterium density to the total deuterium density $n_{D}: f^{+0,+1}=n^{+0,+1} / n_{\text {tot }}$. Figure $3 \mathrm{~b}$ shows the fractional abundance for two different densities. Below $T \approx 1.5 \mathrm{eV}$ almost all deuterium atoms are in the neutral state. For the calculation of $f$ transport effects are neglected and only the balance of ionization and recombination rates is taken into account. The neglected transport effects will lead to a modification of the curves, but the transition from neutral to ionized deuterium is always around $\approx 1.5 \mathrm{eV}$.
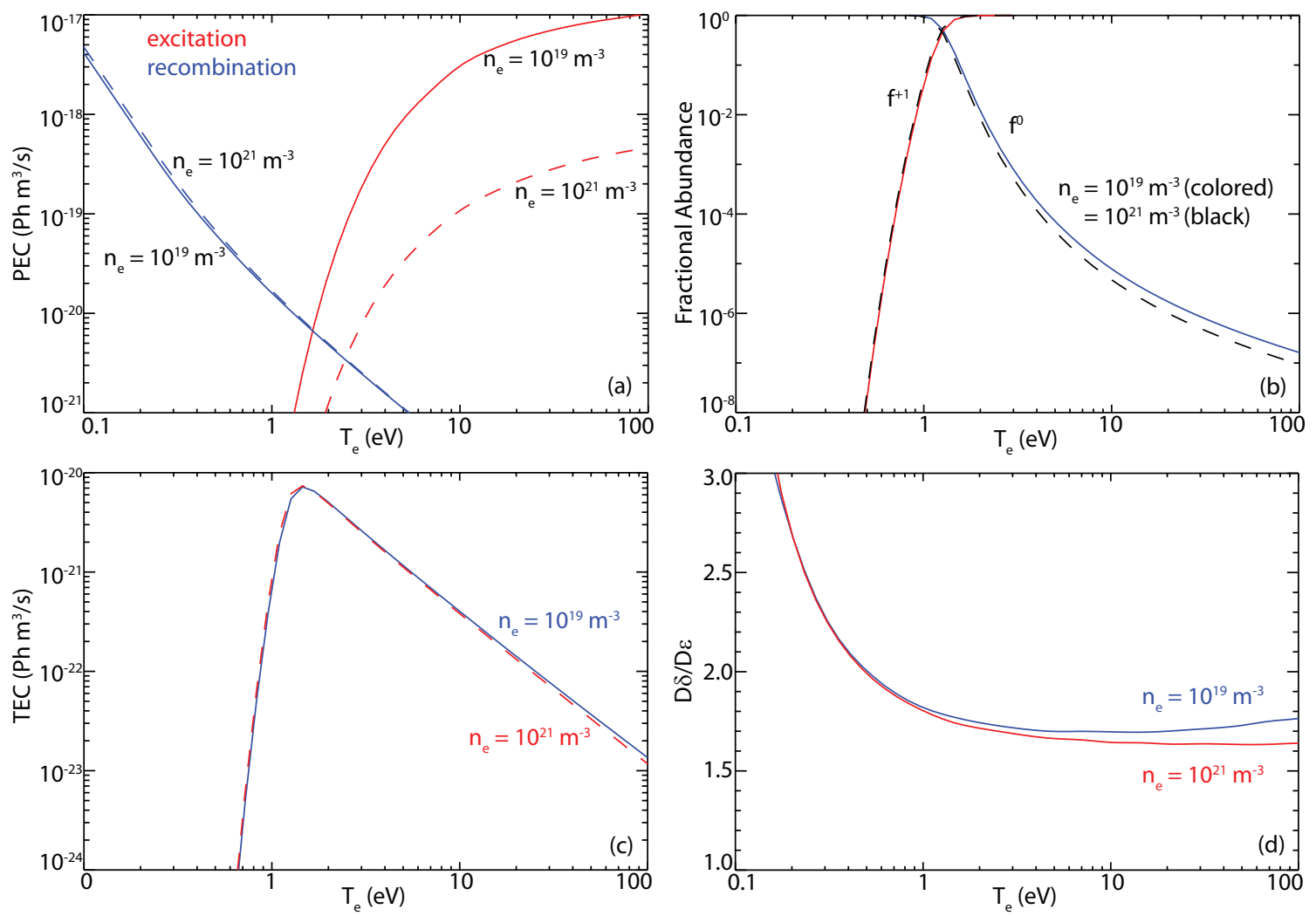

Figure 3. (a) $\mathrm{D}_{\delta}$ photon emission coefficients (PEC) for excitation (red) and recombination (blue), (b) Fractional abundance for neutral (red) and ionized (blue) deuterium, (c) $\mathrm{D}_{\delta}$ total line emission coefficient (TEC) and (d) line emission ratio of $\mathrm{D}_{\delta}$ and $\mathrm{D}_{\epsilon}$. All shown for two different densities.

The expression in the brackets in equation 1 is here called the total line emission coefficient (TEC) and is shown in Figure 3c for two different densities. The TEC peaks at $T_{e} \approx 1.5 \mathrm{eV}$ and drops rapidly when the temperature decreases. This is due to the strong decrease of the ionized deuterium density (Fig. 3b) as the plasma is dominated by recombination (Fig. 3a). Moreover, the TEC does almost not depend on the electron $\ddagger$ Atomic Data and Analysis Structure, http://www.adas.ac.uk/ 
density. The line emission ratio of $\mathrm{D}_{\delta}$ and $\mathrm{D}_{\epsilon}$ is the ratio of the corresponding TECs and given by equation 1 :

$$
\frac{\epsilon_{6 \rightarrow 2}}{\epsilon_{7 \rightarrow 2}}=\frac{\mathrm{TEC}_{D_{\delta}}}{\mathrm{TEC}_{D_{\epsilon}}}
$$

where the electron and total hydrogen densities cancel out. Figure $3 \mathrm{~d}$ shows $\mathrm{D}_{\delta} / \mathrm{D}_{\epsilon}$ for two different densities. The line ratio depends weakly on the electron density and increases strongly at $T_{e}<1 \mathrm{eV}$ when recombination becomes dominant. Due to this strong increase of the line ratio and the flat profile above $1 \mathrm{eV}$ we can determine whether recombination is the dominant process or not.

\subsection{The Two-Point Model and the degree of detachment}

For the conduction limited regime there exists a set of simplified equations which describe the relation between the upstream and target parameters, called the Two-Point Model (TPM) [30]:

$$
\begin{aligned}
T_{t} & =\frac{q_{\text {sep }}^{2}}{n_{\text {sep }}^{2}}\left(\frac{2 \kappa_{0 e}}{7 q_{\text {sep }} L_{c}}\right)^{4 / 7} \frac{2 m_{i}}{\gamma^{2} e^{2}} \frac{\left(1-f_{\text {pow }}\right)^{2}}{\left(1-f_{\text {mom }}\right)^{2}\left(1-f_{\text {conv }}\right)^{4 / 7}} \\
n_{t} & =\frac{n_{\text {sep }}^{3}}{q_{\text {sep }}^{2}}\left(\frac{7 q_{\text {sep }} L_{c}}{2 \kappa_{0 e}}\right)^{6 / 7} \frac{\gamma^{2} e^{3}}{4 m_{i}} \frac{\left(1-f_{\text {mom }}\right)^{3}\left(1-f_{\text {conv }}\right)^{6 / 7}}{\left(1-f_{\text {pow }}\right)^{2}} \\
\Gamma_{t} & =\frac{n_{\text {sep }}^{2}}{q_{\text {sep }}}\left(\frac{7 q_{\text {sep }} L_{c}}{2 \kappa_{0 e}}\right)^{4 / 7} \frac{\gamma e^{2}}{2 m_{i}} \frac{\left(1-f_{\text {mom }}\right)^{2}\left(1-f_{\text {conv }}\right)^{4 / 7}}{\left(1-f_{\text {pow }}\right)}
\end{aligned}
$$

where $n_{\text {sep }}$ and $q_{\text {sep }}$ are the upstream separatrix density and heat flux density entering the SOL, respectively, $L_{c}$ is the connection length from upstream towards the target, $\kappa_{0 e}$ is the electron heat conductivity, $\gamma$ is the sheath heat transmission coefficient and $m_{i}$ is the ion mass. The power loss factor $\left(0<f_{\text {pow }}<1\right)$, the momentum loss factor $\left(0<f_{\text {mom }}<1\right)$ and the convection factor $\left(0<f_{\text {conv }}<1\right)$ accounts for the power and momentum which is lost and the fraction of power which is convected along a field line towards the target, respectively. The simplest case neglecting all loss factors $\left(f_{\text {conv }}=f_{\text {mom }}=f_{\text {pow }}=0\right)$ is called the simple Two-Point Model [31].

Similar to a previous work at JET [32] we use the notation of the degree of detachment (DOD), which is defined as the ratio of the calculated $\left(\Phi_{D^{+}}^{\text {calc }}\right)$ and measured $\left(\Phi_{D^{+}}^{\text {meas }}\right)$ ion flux to the target. According to the TPM the ion flux reaching the divertor target is proportional to the square of the upstream separatrix density $n_{\text {sep }}$ (eq. 5). Furthermore, for Ohmic and L-Mode discharges it has been shown that $n_{u}^{\text {sep }}$ scales approximately linearly with the line integrated plasma density, $\bar{n}_{e}$ [33]. Therefore we

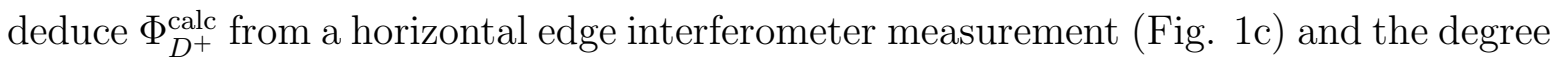
of detachment is then calculated as:

$$
\mathrm{DOD}=\frac{\Phi_{D^{+}}^{\text {calc }}}{\Phi_{D^{+}}^{\text {meas }}}=\frac{C_{1} \cdot\left(n_{u}^{\text {sep }}\right)^{2}}{\Phi_{D^{+}}^{\text {meas }}}=\frac{C \cdot \bar{n}_{e}^{2}}{\Phi_{D^{+}}^{\text {meas }}}
$$


The total ion flux to the entire target is obtained by integrating over all Langmuir probe measurements along the divertor surface:

$$
\begin{aligned}
\Phi_{D^{+}}^{\text {meas }} & =2 \pi \int \Gamma_{D^{+}}(\Delta S) R(\Delta S) d \Delta S \\
& \approx 2 \pi \sum_{\text {iprobes }} \Gamma_{D^{+}}(i) R(i) \cdot\left(\frac{\Delta S(i+1)-\Delta S(i-1)}{2}\right)
\end{aligned}
$$

Where the $\Delta S$ coordinate is the distance from the strike point along the divertor surface in poloidal direction, positive values are in the SOL (see Fig. 2a). It should be noted that the large separation between the third and the second uppermost probe in the inner divertor (Fig. 2a) results in an additional uncertainty of $\Phi_{D^{+}}^{\text {meas }}$.

When the divertor plasma can be described by the simple Two-Point Model, then $\Phi_{D^{+}}^{\text {calc }}=\Phi_{D^{+}}^{\text {meas }}$ and DOD $=1$. The main assumptions of the model are that there is no significant energy and pressure loss along a field line. Simply speaking, when the pressure drops along a field line, hence detachment begins, $\Phi_{D^{+}}^{\text {meas }}$ becomes less than $\Phi_{D^{+}}^{\text {calc }}$ and DOD $>1$. There could be other mechanisms leading to a weaker rise of $\Phi_{D^{+}}^{\text {meas }}$ compared to the TPM scaling such as e.g. a change of the ratio between conducted and convected parallel heat flux or an increase of the radial transport. The linear dependence between the separatrix and the edge density might also change within one discharge during a density ramp. Therefore, for our work we define the onset of detachment when $\Phi_{D^{+}}^{\text {meas }}$ rolls over and use the DOD just as a guide line for the magnitude of detachment.

\section{Evolution of divertor detachment - the three detachment states}

The main observation of this work is that the detachment process can be divided into three different states. In the first state, which we will call the onset state (OS), the first deviation from the simple Two-Point Model scaling happens. In the second one, called the fluctuating state (FS), radiative fluctuations in the inner SOL close to the $\mathrm{X}$-point occur [34]. When these fluctuations vanish, complete detachment over a large target area sets in simultaneously in the inner and outer divertor, giving this third state the name complete detachment state (CDS). Furthermore, the characteristics of these states are a combination of both the inner and outer divertor conditions, meaning that the behavior of both divertors is coupled. These divertor conditions will be described in detail in the following for forward field direction with deuterium fueling (section 3.1), for forward field direction with hydrogen fueling (section 3.2) and for reversed field direction with deuterium fueling (section 3.3).

\subsection{The three detachment states in forward field}

In the following, we describe the evolution of detachment in the inner and outer divertor in forward field direction with deuterium as the main ion species. Representative for all discharges of this series, which are made in forward field, measurements of the discharge 
\# 27100 (Fig. 1, Tb. 1) are shown. The absolute values presented here are only valid for this specific discharge. The qualitative trends of the various parameters during the three detachment states are, however, valid for all discharges made in forward field with deuterium.

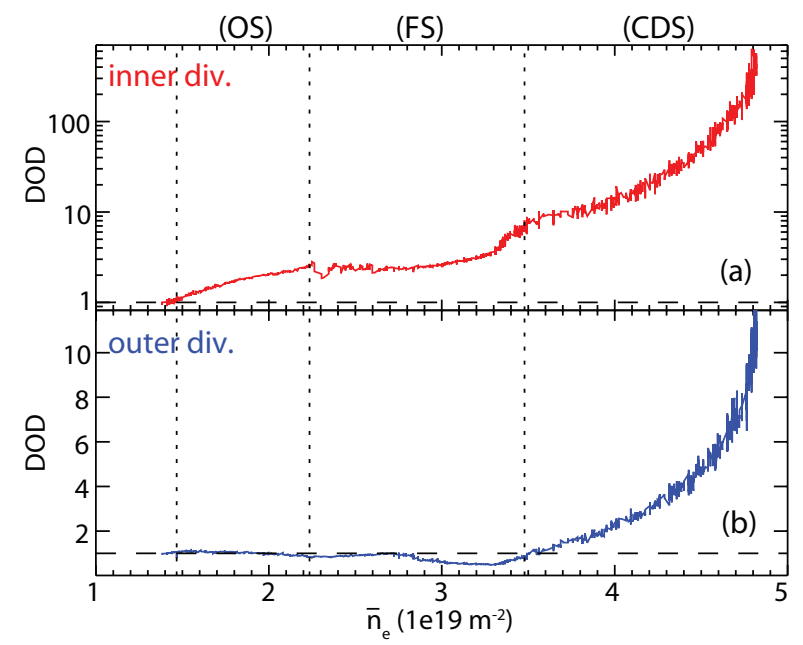

Figure 4. DOD as a function of the line integrated edge plasma density for the inner (a) and outer (b) divertor of discharge \# 27100. Note the logarithmic scale of (a). The three detachment states (OS), (FS) and (CDS) are indicated.

Figure 4 shows the calculated DOD of the inner and outer divertor as a function of the line integrated edge plasma density. To determine the two constants $C$ in Eq. 6 for the inner and outer divertor, the mean values of $\Phi_{D^{+}}^{\text {meas }}(1.8 \mathrm{~s}<t<1.9 \mathrm{~s})$ before the fueling ramp are set equal to $\Phi_{D^{+}}^{\text {calc }}$. At this time the inner divertor is already in a high recycling regime although this is the lowest achievable main plasma density in these density ramp discharges. The measured and calculated temporal evolutions of $\Phi_{D^{+}}$to the inner and outer divertor are shown in Figure 5a,b. From this, the onsets of detachment (when $\Phi_{D^{+}}^{\text {meas }}$ rolls over $)$ of the inner and outer divertors are set to $\bar{n}_{e} \approx 1.5 \cdot 10^{19} \mathrm{~m}^{-2}(t \approx 2 \mathrm{~s})$ and $\bar{n}_{e} \approx 3.3 \cdot 10^{19} \mathrm{~m}^{-2}(t \approx 2.85 \mathrm{~s})$, respectively. Before the onset of the inner divertor detachment at $t<2 \mathrm{~s}, \Phi_{D^{+}}$to the inner divertor is higher than that to the outer divertor. The ratio of both corresponds to the ratio of the constants $C$, see Figure 5a,b: $\Phi_{D^{+}}^{\text {in }} / \Phi_{D^{+}}^{\text {out }}=9.1 / 4.7=1.9$.

The target profiles of $n_{e, t}, T_{e, t}$ and $\Gamma_{D^{+}}$measured by Langmuir probes and the density profiles in the divertor volume measured by the Stark broadening diagnostic (SBD) as a function of time are shown in Figure 6. The $\Delta R$ coordinate for the inner vertical SBD LOS in Figure 6a is the distance from the $\mathrm{X}$-point along a horizontal line, the origin is at the X-point position and negative values are in the inner SOL (see Fig. $2 \mathrm{a})$. Before the onset of the inner divertor detachment $(t<2 \mathrm{~s})$ peak ion fluxes of $\Gamma_{D^{+}} \approx 2 \cdot 10^{22} \mathrm{~m}^{-2} \mathrm{~s}^{-1}$ close to the inner strike point and $\Gamma_{D^{+}} \approx 1.5 \cdot 10^{22} \mathrm{~m}^{-2} \mathrm{~s}^{-1}$ close to the outer strike point are measured, respectively. Their ratio of $\Gamma_{D^{+}}^{i n} / \Gamma_{D^{+}}^{o \text { ou }}=1.3$ is more symmetric than the total ion flux ratio of $\Phi_{D^{+}}^{\text {in }} / \Phi_{D^{+}}^{\text {out }}=1.9$. This means that the inner 


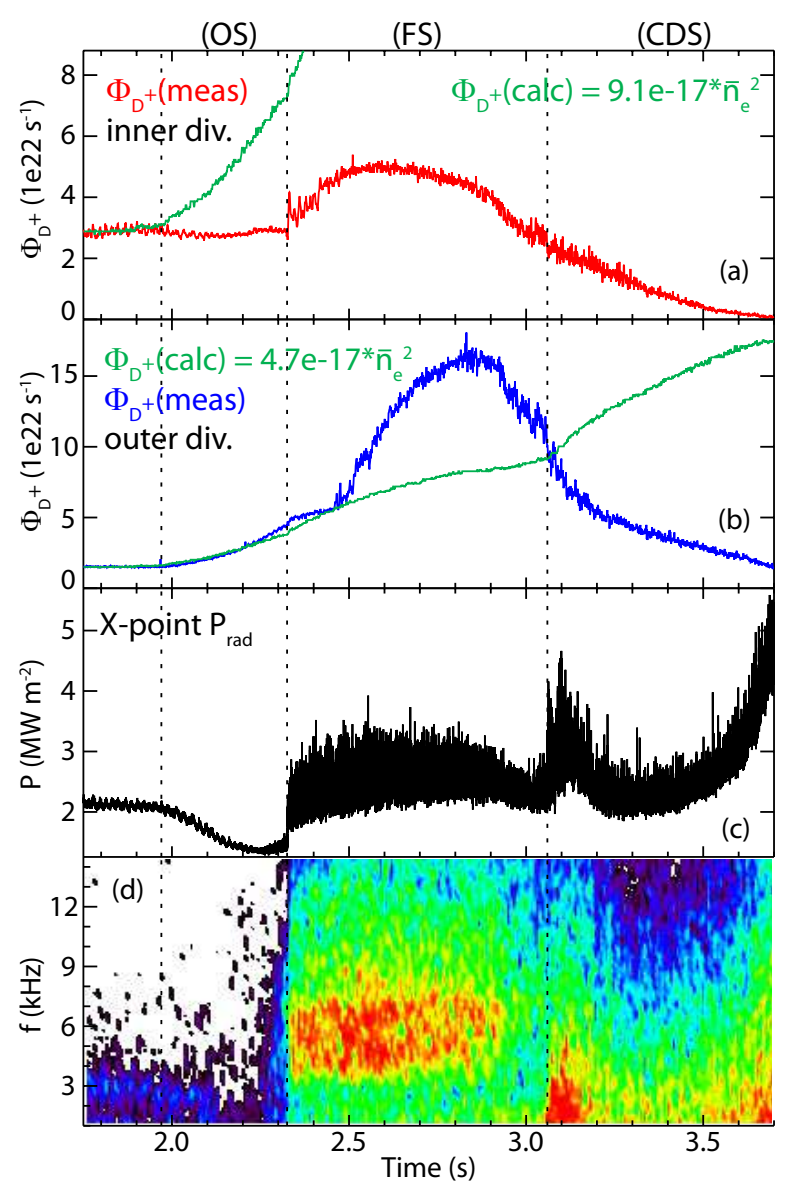

Figure 5. Calculated and measured total ion flux to the inner (a) and outer target (b), line integrated radiated power (c) measured by an AXUV diode (orange chord in Figure 2b) and its power spectrum (d) of discharge \# 27100. The three detachment states (OS), (FS) and (CDS) are indicated.

ion flux profile is broader than the outer one which indicates that the inner divertor is already in a higher recycling regime, as mentioned above. Before the onset of detachment $(t<2 \mathrm{~s})$ the electron density in the divertor volume is below the measurement range of $n_{e}>4 \cdot 10^{19} \mathrm{~m}^{-3}$ of the SBD in both the inner and outer divertor. This is expected as the density at the targets, measured by probes, is below $1.5 \cdot 10^{19} \mathrm{~m}^{-3}$, which corresponds to a density at the recycling zone of $n_{e, V} \approx 2 \cdot n_{e, t} \approx 3 \cdot 10^{19} \mathrm{~m}^{-3}$ (e.g. Ref. [30]).

3.1.1. The onset of detachment state The start of this state is defined by the first deviation from the TPM scaling, which occurs in the inner divertor at $\bar{n}_{e} \approx 1.5 \cdot 10^{19} \mathrm{~m}^{-2}$ (Fig. 4a) or at $t \approx 2 \mathrm{~s}$ (Fig. 5a). Namely $\Phi_{D^{+}}^{\text {meas }}$ becomes less than the TPM scaling and the DOD exceeds unity.

Inner divertor During this state, the ion flux close to the inner strike point, $\Delta S \approx 1 \mathrm{~cm}$ rolls over and drops to $\Gamma_{D^{+}} \approx 1 \cdot 10^{22} \mathrm{~m}^{-2} \mathrm{~s}^{-1}$ with increasing upstream density (Fig. 6h). This is also visible in the total ion flux to the inner divertor which further deviates from 

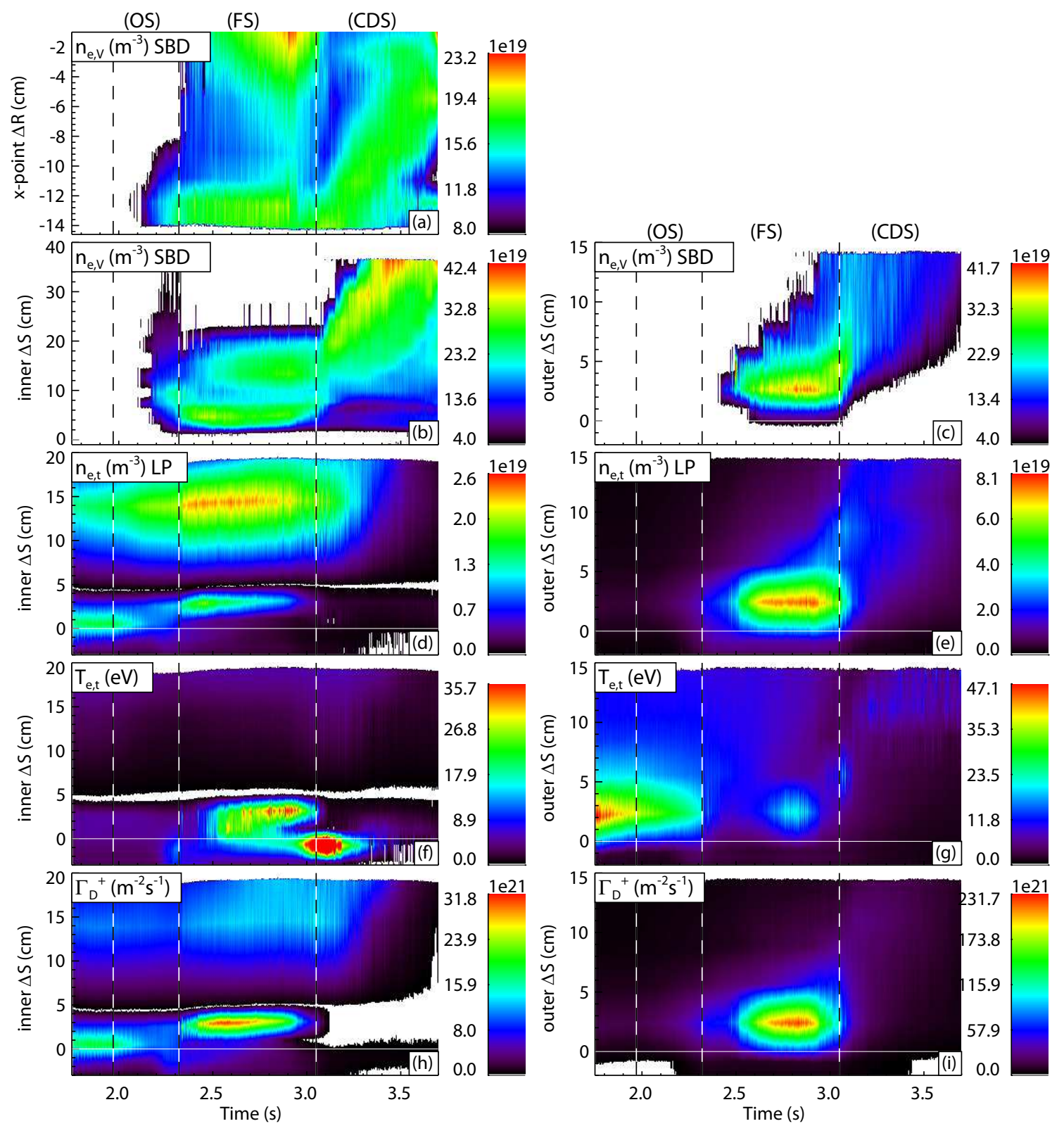

Figure 6. Horizontal (a) and vertical (b,c) $n_{e, V}$ profile in the divertor volume and $n_{e, t}(\mathrm{~d}, \mathrm{e}), T_{e, t}(\mathrm{f}, \mathrm{g})$ and $\Gamma_{D^{+}}(\mathrm{h}, \mathrm{i})$ target profiles in the inner and outer divertor, respectively, of discharge \# 27100. The three detachment states (OS), (FS) and (CDS) are indicated.

the TPM scaling (Fig. 5a) and the DOD increases (Fig. 4a). Also $n_{e, t}$ close to the strike point decreases with increasing upstream density, but, in contrast, increases in the far SOL at $\Delta S \approx 14 \mathrm{~cm}$ up to $n_{e, t} \approx 2.3 \cdot 10^{19} \mathrm{~m}^{-3}$ at the end of the onset state (Fig. $6 \mathrm{~d}$ ). This is consistent with the radiation distribution measured by foil bolometry, shown in Figure 7. The radiation is higher in the inner far SOL than close to the strike point. In addition, the electron density in the volume increases up to $n_{e, v} \approx 2.3 \cdot 10^{20} \mathrm{~m}^{-3}$ (Fig. $6 \mathrm{~b})$. All in all, this indicates that the plasma is partially detached from the inner strike 


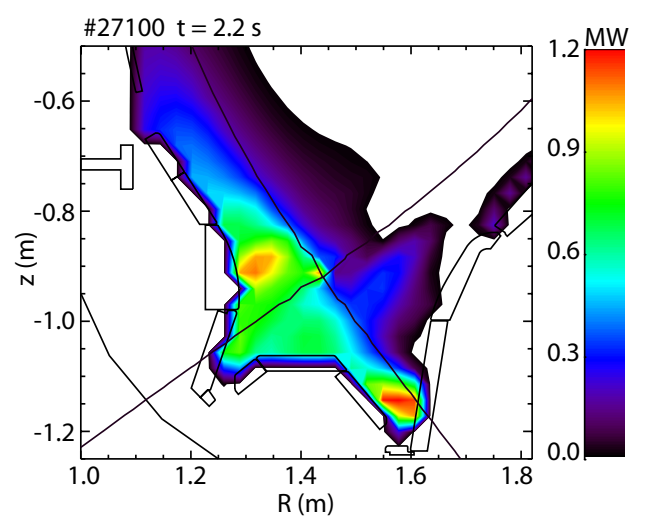

Figure 7. Total radiation distribution from foil bolometry in the divertor at $t=2.2 \mathrm{~s}$ during the onset state for discharge \# 27100 .

point region at the end of this state.

Outer divertor The outer divertor is still attached, in the conduction limited regime and follows the simple TPM (DOD =1) throughout this state (Figs. 4b, 5b). With increasing upstream density, the maximum of $\Gamma_{D^{+}}$and $n_{e, t}$ increases during this state up to $4 \cdot 10^{22} \mathrm{~m}^{-2} \mathrm{~s}^{-1}$ and $1.4 \cdot 10^{19} \mathrm{~m}^{-3}$, respectively, while $T_{e, t}$ drops to $25 \mathrm{eV}$ (Fig. $6 \mathrm{i}, \mathrm{e}, \mathrm{g}$ ). The electron density in the volume stays below $4 \cdot 10^{19} \mathrm{~m}^{-3}$ (Fig. 6c).

3.1.2. The fluctuating detachment state This state is defined by the appearance of radiative fluctuations which are situated close to the X-point in the inner SOL. During this state, the core plasma fueling becomes less efficient. Although the amount of the fueling gas is steadily increased, the core plasma density seems to saturate (Fig. 1c, $t \approx 2.9 \mathrm{~s}$ ). The characteristics of these fluctuations and the evolution of the divertor plasma parameters during this state are described in the following.

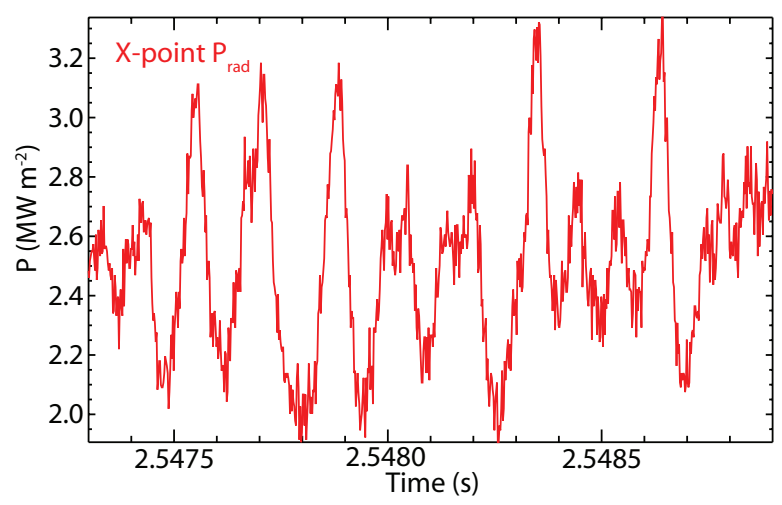

Figure 8. Temporal evolution of the radiative X-point fluctuations measured by an AXUV diode (orange chord in Figure 2b) of discharge \# 27100. 
The X-point fluctuations The transition to this state is determined with a sudden onset of a fluctuation band of $f \approx 5.5 \mathrm{kHz}$ (Fig. $5 \mathrm{c}, \mathrm{d}$ ), measured with the AXUV diodes, which is observed for the first time. The spectrogram was derived with a short-time Fourier transform with a sample window size of $n_{F F T}=4096$ (the choice of $n_{F F T}$ was found to have no significant impact on the power spectra). The width of this fluctuation band is $\Delta f \approx 3 \mathrm{kHz}$. Figure 8 shows a zoom of an AXUV time trace measured with the orange chord in Figure $2 \mathrm{~b}$. The mean value of the radiated power is $\approx 2.5 \mathrm{MW} \mathrm{m}^{-2}$ and its standard deviation is $\approx 0.3 \mathrm{MW} \mathrm{m}^{-2}$. There is currently no other diagnostic available measuring with such a high sampling rate in the according region in order to trace these fluctuations back to the electron temperature or to the density or to a combination of both.
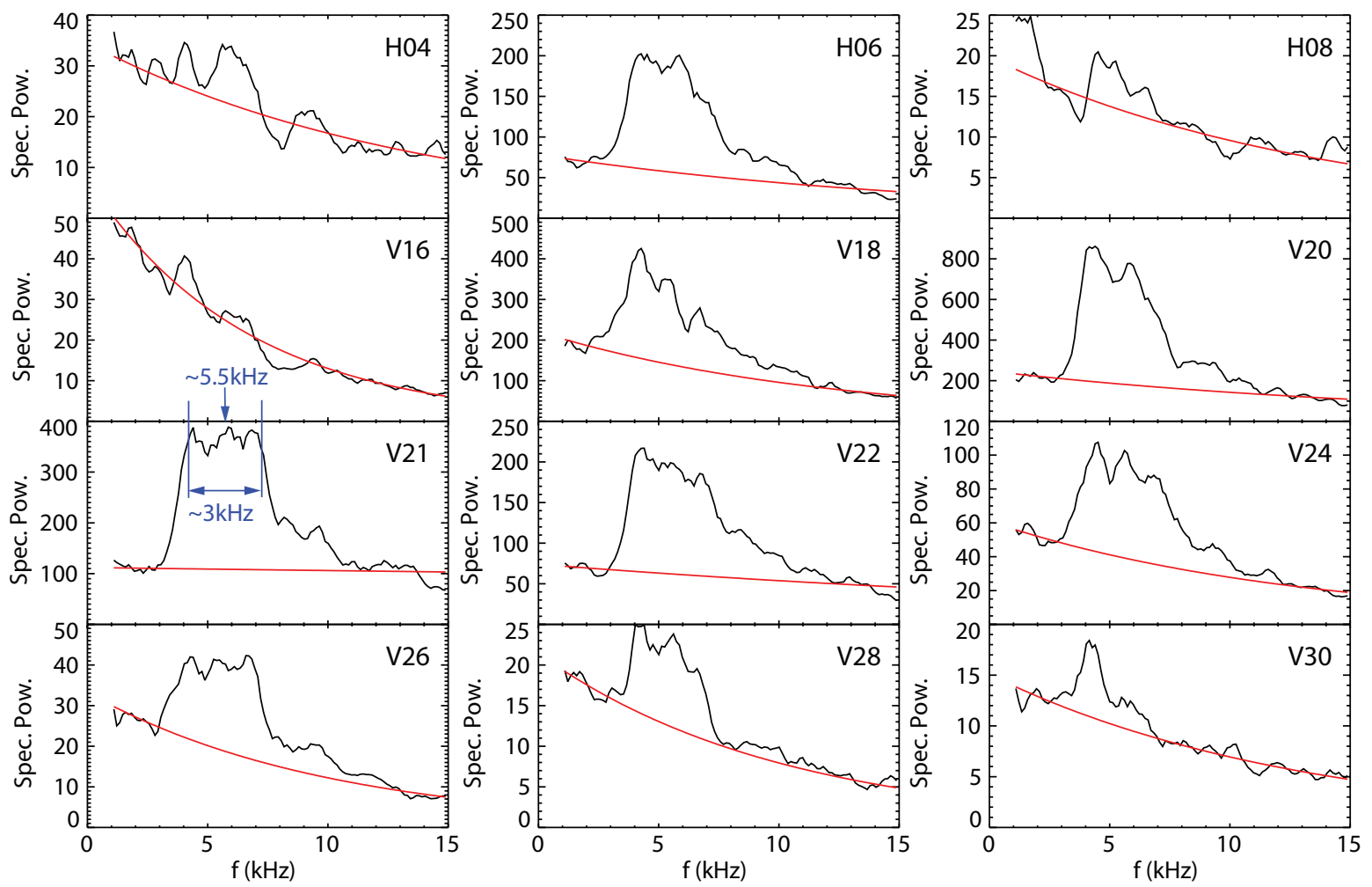

Figure 9. Spectral power versus frequency for various AXUV diodes (black) and fit of an exponential function on the background (red) of discharge \# 27100. The positions of the diodes are shown in Figures2b and 10.

Figure 9 shows the average frequency power spectra of the fluctuations for various diodes. First the spectrogram was made as described above and then averaged over the time interval from 2.4 to $2.6 \mathrm{~s}$. The width and the mean frequency are indicated for a specific diode. With all AXUV channels the position of these fluctuations can be located. The strength of the fluctuations is derived by integrating over the spectrum after subtracting the background level. The background is due to electronic noise, which is at these rather low frequencies determined by flicker noise and can thus be approximated with an exponential function. The fit of an exponential function on the 
spectrum is also shown in Figure 9. The exponential function is a sufficient assumption for the background as we are interested in the relative strength of the various signals and not in absolute numbers. The resulting fluctuation strength for all diodes is shown in Figure 10, where the color-code represents the strength. It can be seen that the radiative fluctuations are located close to the X-point in the inner SOL.

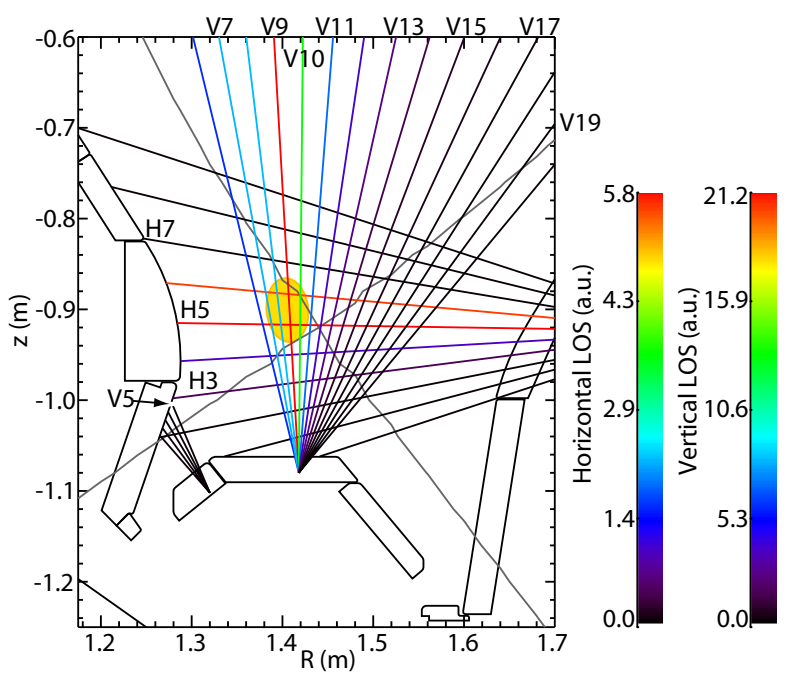

Figure 10. Intensity of the fluctuations for all AXUV channels of discharge \# 27100. The color-code represents the strength and the location of the fluctuations is indicated by the yellow circle.

Inner divertor At the start of the fluctuating state, there is a sudden increase of $\Phi_{D^{+}}$ (Fig. 5a) and $\Gamma_{D^{+}}$as well as an abrupt shift of the peak $\Gamma_{D^{+}}$position away from the strike point from $\Delta S \approx 1 \mathrm{~cm}$ to $\Delta S \approx 3 \mathrm{~cm}$ (Fig. 6h). During this state, the ion flux at this position first increases up to $\Gamma_{D^{+}} \approx 3.2 \cdot 10^{22} \mathrm{~m}^{-2} \mathrm{~s}^{-1}$ and then rolls over at $t \approx 2.6 \mathrm{~s}$. The target electron density at the strike point region shows the same shift and also rolls over, see Figure $6 \mathrm{~d}$. In contrast, the electron temperature at this narrow region $(\Delta S \approx 3 \mathrm{~cm})$ steadily increases up to about $T_{e, t} \approx 36 \mathrm{eV}$ (Fig. 6f) while the pressure there remains almost constant. The ion flux in the far SOL at $\Delta S>14 \mathrm{~cm}$ increases throughout this state, whereas $n_{e, t}$ in this region, which is higher than at the strike point, rolls over during this state. The temperature in this region remains low, $T_{e, t} \approx 4 \mathrm{eV}$.

At the transition to the fluctuating state the peak density in the volume, $n_{e, V}$, shifts from $\Delta S \approx 10 \mathrm{~cm}$ to $\Delta S \approx 4 \mathrm{~cm}$ (Fig. $6 \mathrm{~b}$ ). Associated with the second roll over of $\Gamma_{D^{+}}$ and $n_{e, t}$ at the strike point region (Fig. $6 \mathrm{~h}, \mathrm{~d}$ ) a region of high density $\approx 2.5 \cdot 10^{20} \mathrm{~m}^{-3}$ develops in the volume of the inner far SOL $(\Delta S \approx 15 \mathrm{~cm}$, Fig. $6 \mathrm{~b})$. This density is an order of magnitude higher than $n_{e, t}$ and $\bar{n}_{e}$. In the following these regions are called high density fronts. The position can be verified with the vertical $n_{e, V}$ measurements (Fig. 6a, $\Delta R=-14 \mathrm{~cm}$ ) which are from the leftmost LOS (Fig. 2a) close to the target. In addition, a second high density front starts to develop in the inner SOL close to the 


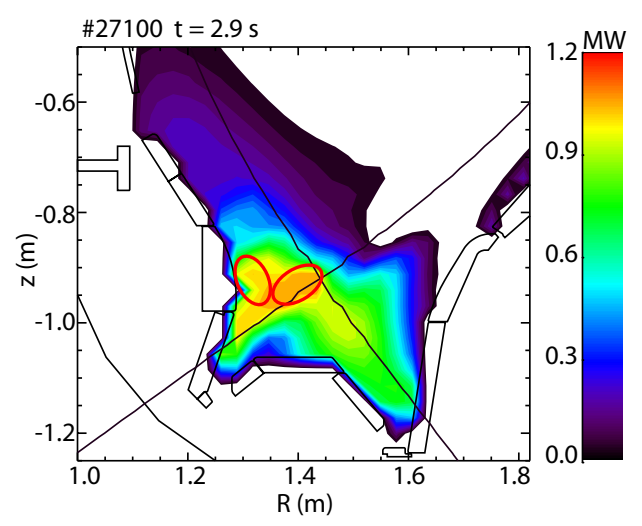

Figure 11. Total radiation distribution from foil bolometry in the divertor at $t=2.9 \mathrm{~s}$ during the fluctuating detachment state for discharge \# 27100. The estimated positions of the high density fronts in the inner divertor are indicated with the red circles.

$\mathrm{X}$-point (Fig. 6a, $\Delta R=-2 \mathrm{~cm}$ ). The region where the $\mathrm{SBD}$ diagnostic measures the density corresponds to regions where the radiation is highest $\left(P_{r a d} \propto n_{e}^{2}\right)$. Therefore, the total radiation distribution measured by foil bolometry can be used as an approximation of the density distribution. The radiation distribution for $t=2.9 \mathrm{~s}$ is shown in Figure 11 and the approximated positions of the high density fronts are indicated. It is also possible, according to the radiation measurement, that this is just one density front rather than two. In any case, however, the density front in the inner divertor expands into the inner far SOL at $\Delta S \approx 15 \mathrm{~cm}$, consistent with the increase of the ion flux and target electron density in this region. With increasing upstream density, first the spatial extent of the X-point high density front grows into the inner SOL and $n_{e, V}$ rises up to $\approx 2.5 \cdot 10^{20} \mathrm{~m}^{-3}$ and then rolls over (Fig. 6a).

Outer divertor During the fluctuating state the ion flux and the electron density at the outer target first increase and then roll over as in the inner one (Fig. 6e,i). The peak positions of $\Gamma_{D^{+}}$and $n_{e, t}$ remain constant during the discharge. Moreover, $\Phi_{D^{+}}^{\text {meas }}$ starts to surpass the TPM scaling, $\Phi_{D^{+}}^{\text {meas }}>\Phi_{D^{+}}^{\text {calc }}$ (Figure $5 \mathrm{~b}$ ), which in the following will be called flux enhancement. Consequently the DOD drops below 1, see Figure 4b. Contrary to the inner target, $T_{e, t}$ also increases while $\Gamma_{D^{+}}$and $n_{e, t}$ increase and then rolls over (Fig. 6g). This simultaneous increase of $T_{e, t}$ and $n_{e, t}$ leads to a rise of the outer divertor pressure by $\approx 100 \%$.

In line with this, the density in the outer divertor volume close to the strike point rises by more than an order of magnitude up to $n_{e, V} \approx 4 \cdot 10^{20} \mathrm{~m}^{-3}$ (Fig. $6 \mathrm{c}$ ), reaching its maximum after $\Gamma_{D^{+}}$and $n_{e, t}$ rolled over. As the spatial extent of the Stark diagnostic in the outer divertor is limited to $\Delta S<15 \mathrm{~cm}$ (Fig. 2a) it cannot be verified if there exists a high density front in the far outer SOL or X-point region similar to the one in the inner divertor. 
3.1.3. The complete detachment state The beginning of this state is defined when the $\mathrm{X}$-point fluctuations disappear (Fig. 5d). With the transition from the fluctuating state to the complete detachment state, the core plasma fueling becomes more efficient again. This can be seen in the time traces of the line integrated plasma density and the applied fueling gas puff (Fig. 1c, $t \approx 3 \mathrm{~s}$ ). The plasma density increases faster at this point while the fueling rate remains constant. In the following, the evolution of the divertor plasma parameters are described in detail and the occurrence of volume recombination will be discussed.

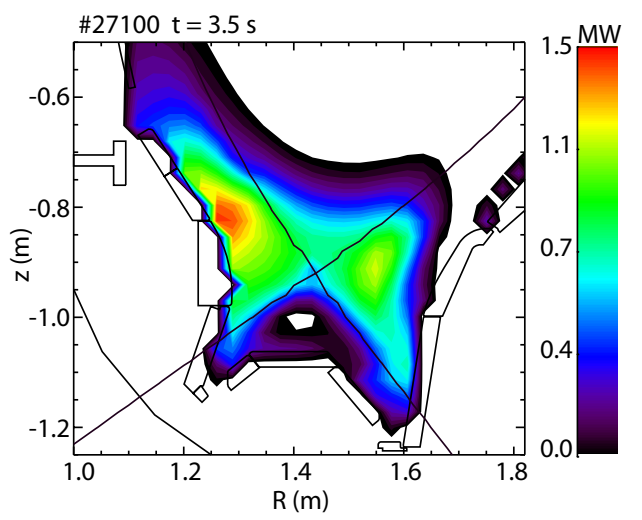

Figure 12. Total radiation distribution from foil bolometry in the divertor at $t=3.5 \mathrm{~s}$ during the complete detachment state for discharge \# 27100 .

Inner divertor With the start of this state $n_{e, t}, T_{e, t}$ and $\Gamma_{D^{+}}$at the strike point region are reduced by more than an order of magnitude (Fig. 6d,f,h). Also the high density front at the strike point region disappears and moves along the field lines towards and even above the X-point. With the horizontal and vertical $n_{e, V}$ measurements, the motion of the high density front can be monitored. At the beginning the density front is located at $\Delta S=15 \mathrm{~cm}$ (Fig. $6 \mathrm{~b}$ ) and close to the target at $\Delta R=-14 \mathrm{~cm}$ (Fig. 6a). Then the front moves in horizontal as well as in vertical direction until it is close to the X-point at $\Delta R=-2 \mathrm{~cm}$ and $\Delta S=38 \mathrm{~cm}$ (which are the uppermost horizontal LOS, Fig. 2a, being above the $\mathrm{X}$-point). The final position of the density front, at $t=3.5 \mathrm{~s}$, is consistent with the radiation distribution (Fig. 12), which peaks well above the X-point. We call here the situation when the target values $n_{e, t}, T_{e, t}$ and $\Gamma_{D^{+}}$and also the electron density in the volume, $n_{e, V}$, almost vanish at the strike point region complete detachment $\S$. Thus the name for this third detachment state. It should be noted here, that there is a short increase of $T_{e, t}$ close to the strike point just after the beginning of this state when the density front starts to move towards the X-point $(t \approx 3 \mathrm{~s}$, Fig. $6 \mathrm{f})$. The uncertainty in the determination of the strike point position is $\approx 1 \mathrm{~cm}$. It is therefore not possible to clarify if this short increase of $T_{e, t}$ occurs in the SOL or in the private flux region.

$\S$ The reader should not be confused with the definition of total detachment based on the DOD [32] 
It is also unlikely to be a measurement error of the Langmuir probes as this feature is observed in every discharge of this density ramp series.
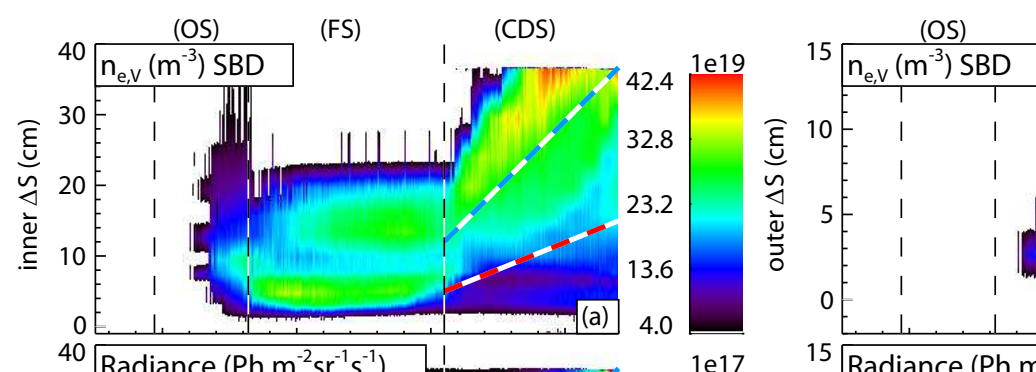

(FS)

(CDS)
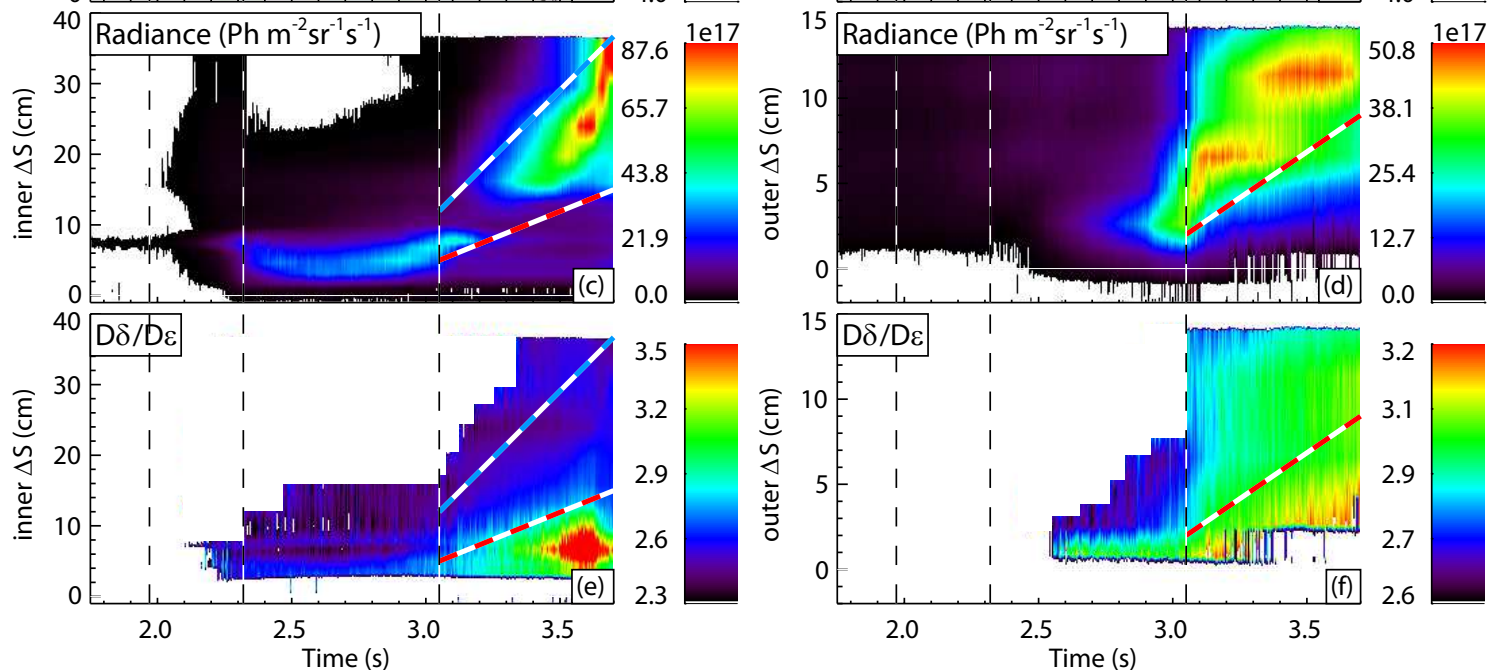

Figure 13. Profiles of $n_{e, V}(\mathrm{a}, \mathrm{b})$, the $\mathrm{D}_{\delta}$ emissivity $(\mathrm{c}, \mathrm{d})$ and the line ratio $\mathrm{D}_{\delta} / \mathrm{D}_{\epsilon}(\mathrm{e}, \mathrm{f})$ in the inner and outer divertor volume, respectively of discharge \# 27100. The three detachment states (OS), (FS) and (CDS) are indicated. See text for further explanation.

Outer divertor At the transition from the fluctuating state to the complete detachment state, $\Phi_{D^{+}}$in the outer divertor becomes smaller than the TPM scaling and the DOD exceeds unity (Figs. 5b, 4b). At this point, $\Gamma_{D^{+}}$at the outer target is already strongly reduced by two orders of magnitude with respect to its maximum value (Fig. 6i). Also the density and the temperature at the target close to the strike point region $(\Delta S<5 \mathrm{~cm})$ have reached low values of $n_{e, t} \leq 2 \cdot 10^{19} \mathrm{~m}^{-3}$ and $T_{e, t} \leq 5 \mathrm{eV}$. Moreover, the high density front in the outer strike point region rapidly moves out of the spectroscopic observation area $(\Delta S<15 \mathrm{~cm})$ at the beginning of this state (Fig. 6c). In the observed region the plasma is therefor completely detached from the outer target, confirmed by the measurement of the total radiation (Fig. 12), which peaks close to the X-point. It is remarkable that the complete detachment starts simultaneously at the inner and outer strike point region. This has not yet been observed elsewhere.

Evidence for volume recombination and low divertor temperatures The density in the divertor volume, the emissivity of $\mathrm{D}_{\delta}$ and the line ratio $\mathrm{D}_{\delta} / \mathrm{D}_{\epsilon}$ is shown in Figure 13. 
First it can be seen that the $\mathrm{D}_{\delta}$ emission increases when the density has decreased, in the inner as well as in the outer divertor. In other words, when the density front has moved towards the X-point (above the blue dashed line in Fig. 13a), the line emission increases between the density front and the target (below the blue dashed line in Fig. 13c). The total line emission coefficient, shown in Figure 3c, increases with decreasing temperature and reaches its maximum at $T_{e} \approx 1.5 \mathrm{eV}$. This peak corresponds to the transition from an ionization to a recombination dominated plasma (sec. 2.3) and the temperature is approximately $\approx 1.5 \mathrm{eV}$. Thereafter, when the emission decreases, the line ratio of $\mathrm{D}_{\delta} / \mathrm{D}_{\epsilon}$ starts to increase (below the red dashed line in Fig. 13c,d,e,f). From Figure $3 \mathrm{~d}$ it can be seen that the line ratio increases when recombination becomes dominant and the temperature is below $1 \mathrm{eV}$. This is consistent with the drop of the total line emission coefficient below $T_{e}=1 \mathrm{eV}$ (Fig. 3c). As these are line integrated measurements, we do not aim at deducing absolute temperatures out of the $\mathrm{D}_{\delta} / \mathrm{D}_{\epsilon}$ ratio. However, we can assume that the radiation of $\mathrm{D}_{\delta}$ and $\mathrm{D}_{\epsilon}$ is emitted from the same region and draw qualitative conclusions. Namely that, in the inner and outer divertor volume, recombination becomes dominant and the electron temperature is $T_{e}<1 \mathrm{eV}$ at times and regions below the red dashed lines in Figure 13.

\subsection{Detachment in hydrogen}

To disentangle isotope effects, several discharges of the density ramp series were performed with hydrogen as main ion species (see Table 1). The divertor plasma undergoes the same three detachment states in hydrogen as in deuterium. The characteristics of the different detachment states described above qualitatively hold also for a hydrogen plasma with the following main quantitative differences. The ratio of the total ion flux to the inner and outer divertor before the beginning of the fueling ramp $\left(t<1.9 \mathrm{~s}\right.$ ) is $\Phi_{D^{+}}^{\text {in }} / \Phi_{D^{+}}^{\text {out }}=1.6$ (Fig. 14a,b), which is more symmetric compared to the deuterium fueled case $\left(\Phi_{D^{+}}^{\text {in }} / \Phi_{D^{+}}^{\text {out }}=1.9\right)$. In addition, the radiative X-point fluctuations during the fluctuating detachment state have a frequency of $f \approx 8 \mathrm{kHz}$ in the hydrogen discharges, see Figure 14d. This frequency is roughly the one in deuterium times the square root of the mass ratio of both species:

$$
8 \mathrm{kHz} \approx \sqrt{\frac{m_{D}}{m_{H}}} \cdot 5.5 \mathrm{kHz}=1.4 \cdot 5.5 \mathrm{kHz}=7.7 \mathrm{kHz}
$$

\subsection{The three detachment states in reversed field}

In this section we describe the evolution of the divertor plasma when $B_{t}$ and $I_{p}$ are reversed and compare it to the forward field case. Representative for all discharges of the series which are made in reversed field we show measurements of the discharge \# 27284 (Table 1). The discharge \# 27101 in forward field has the same heating power as this discharge \# 27284 in reversed field. But in discharge \# 27101 we observed divertor plasma oscillations [34], thus we do not compare this pair of discharges here. When these discharges were carried out, the foil bolometry was not available. Therefore, 


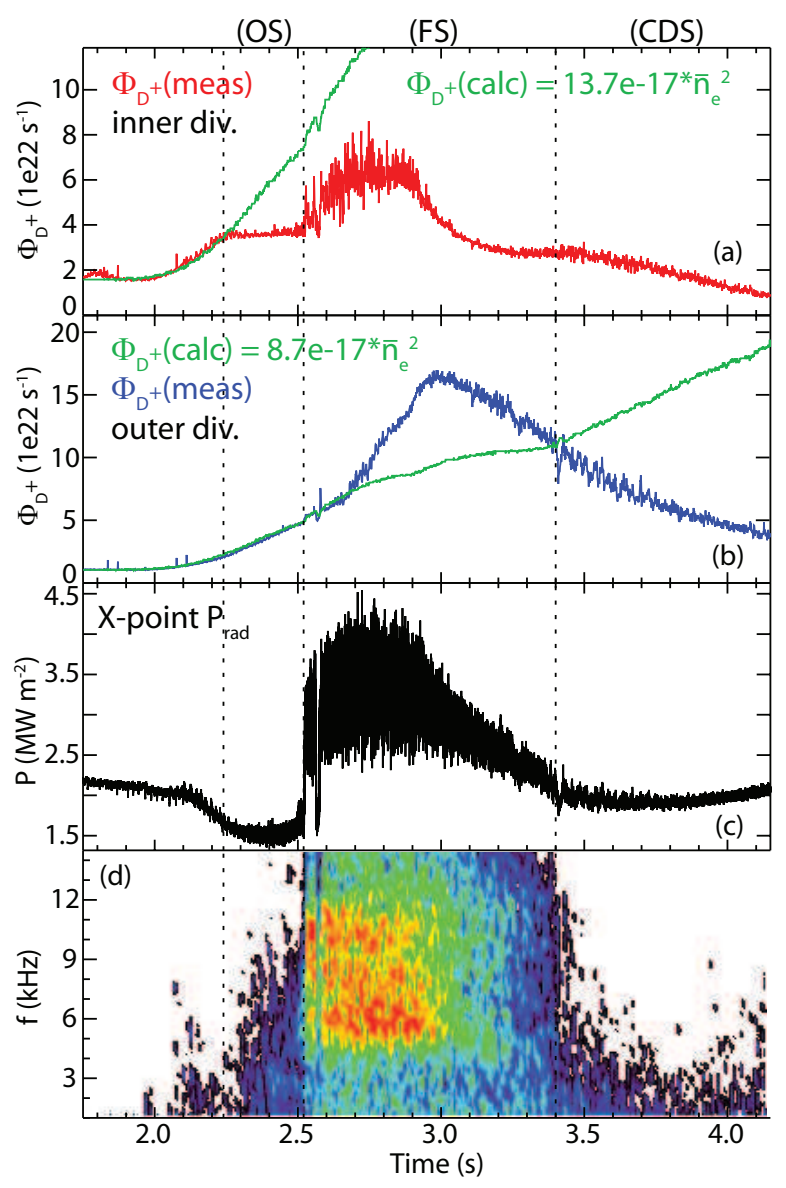

Figure 14. Calculated and measured total ion flux to the inner (a) and outer target (b), line integrated radiated power (c) measured by an AXUV diode (orange chord in Figure 2b) and its power spectrum (d) of discharge \# 27362. The three detachment states (OS), (FS) and (CDS) are indicated.

no measurements of the radiation distribution in the divertor can be shown here. As in forward field, there are three different states during the evolution of detachment. The qualitative development of the target parameters $n_{e, t}, T_{e, t}$ and $\Gamma_{D^{+}}$in the inner, respectively outer target in reversed field is rather comparable to the outer, respectively the inner target in forward field. Whereas the density in the divertor volume evolves quite similar in both field directions.

Figure 15 shows the DOD as a function of the line integrated edge plasma density of the inner and outer divertor and the corresponding measured and calculated temporal evolutions of $\Phi_{D^{+}}$are shown in Figure 16. The onsets of detachment (roll over of $\Phi_{D^{+}}$) of the inner and outer divertors are therefore at $t \approx 2.4 \mathrm{~s}\left(\bar{n}_{e} \approx 2.1 \cdot 10^{19} \mathrm{~m}^{-2}\right)$ and $t \approx 2.5 \mathrm{~s}$ $\left(\bar{n}_{e} \approx 2.5 \cdot 10^{19} \mathrm{~m}^{-2}\right)$, respectively. Before the start of the fueling ramp, $t<1.9 \mathrm{~s}$, the total ion flux to the outer target is larger than to the inner target, $\Phi_{D^{+}}^{\text {out }} / \Phi_{D^{+}}^{\text {in }}=3.6$ (Fig. $16 \mathrm{a}, \mathrm{c})$. This ratio is less symmetric compared to the forward field case and, contrary to forward field, in favor of the outer divertor.

Figure 17 shows the inner and outer target profiles of $n_{e, t}, T_{e, t}$ and $\Gamma_{D^{+}}$as well as 


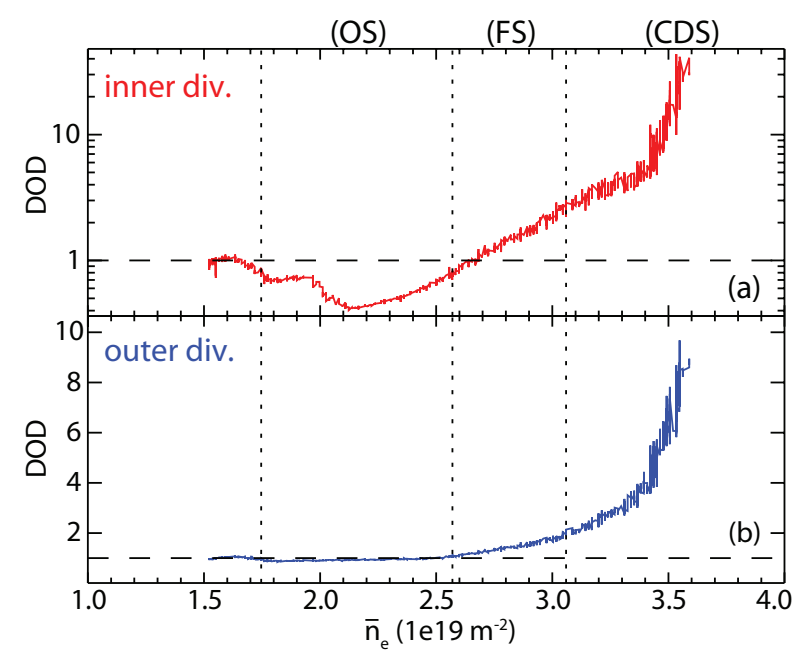

Figure 15. DOD as a function of the line integrated edge plasma density for the inner (a) and outer (b) divertor of discharge \# 27284. Note the logarithmic scale of (a). The three detachment states (OS), (FS) and (CDS) are indicated.

the density measurements in the inner and outer divertor volume. Before the start of the fueling ramp, peak ion fluxes of $\Gamma_{D^{+}} \approx 8 \cdot 10^{21} \mathrm{~m}^{-2} \mathrm{~s}^{-1}$ and $\Gamma_{D^{+}} \approx 5.2 \cdot 10^{22} \mathrm{~m}^{-2} \mathrm{~s}^{-1}$ close to the inner and outer strike point are measured, respectively.

3.3.1. The onset of detachment state As in forward field, the start of the onset state is defined by the first deviation from the TPM scaling. This happens, similar to forward field, in the inner divertor. Contrary to forward field, the X-point fluctuations appear already in this state. Their amplitude grows during this state until, at the end of this state, its maximum is reached.

Inner divertor Contrary to forward field, the measured total ion flux increases more strongly compared to the TPM scaling (Fig. 16a). Thus, the flux enhancement occurs now in the inner divertor and the DOD falls below unity (Fig. 15a). During this state $\Gamma_{D^{+}}$and $n_{e, t}$ increase up to $\Gamma_{D^{+}} \approx 5 \cdot 10^{22} \mathrm{~m}^{-2} \mathrm{~s}^{-1}$ and $n_{e, t} \approx 6 \cdot 10^{19} \mathrm{~m}^{-3}$, respectively, and then roll over. The target temperature first decreases with increasing $n_{e, t}$ and $\Gamma_{D^{+}}$ and increases when $n_{e, t}$ and $\Gamma_{D^{+}}$roll over (Fig. 17d,f,h). Associated with the roll over, a high density front of $n_{e, V} \approx 2 \cdot 10^{20} \mathrm{~m}^{-3}$ develops in the SOL at $\Delta S \approx 7 \mathrm{~cm}$, see Figure 17b. It should be noted that, while $\Gamma_{D^{+}}$increases between $t=2.1 \mathrm{~s}$ and $t=2.3 \mathrm{~s}$, there seems to be a shift of $\Gamma_{D^{+}}$back to $\Delta S \approx 3 \mathrm{~cm}$ at $t=2.2 \mathrm{~s}$ (Fig. 17h). But it is more likely that the peak of the ion flux profile moves between two probe positions in this case.

Outer divertor The measured $\Phi_{D^{+}}$follows the TPM scaling throughout this state and the DOD is 1 , see Figures $15 \mathrm{~b}$ and $16 \mathrm{~b}$. The peak position of $\Gamma_{D^{+}}$and $n_{e, t}$ remains constant during the discharge (Fig. $17 \mathrm{e}, \mathrm{i}) . \Gamma_{D^{+}}$rises up to $\approx 1.6 \cdot 10^{23} \mathrm{~m}^{-2} \mathrm{~s}^{-1}$ and $n_{e, t}$ up 


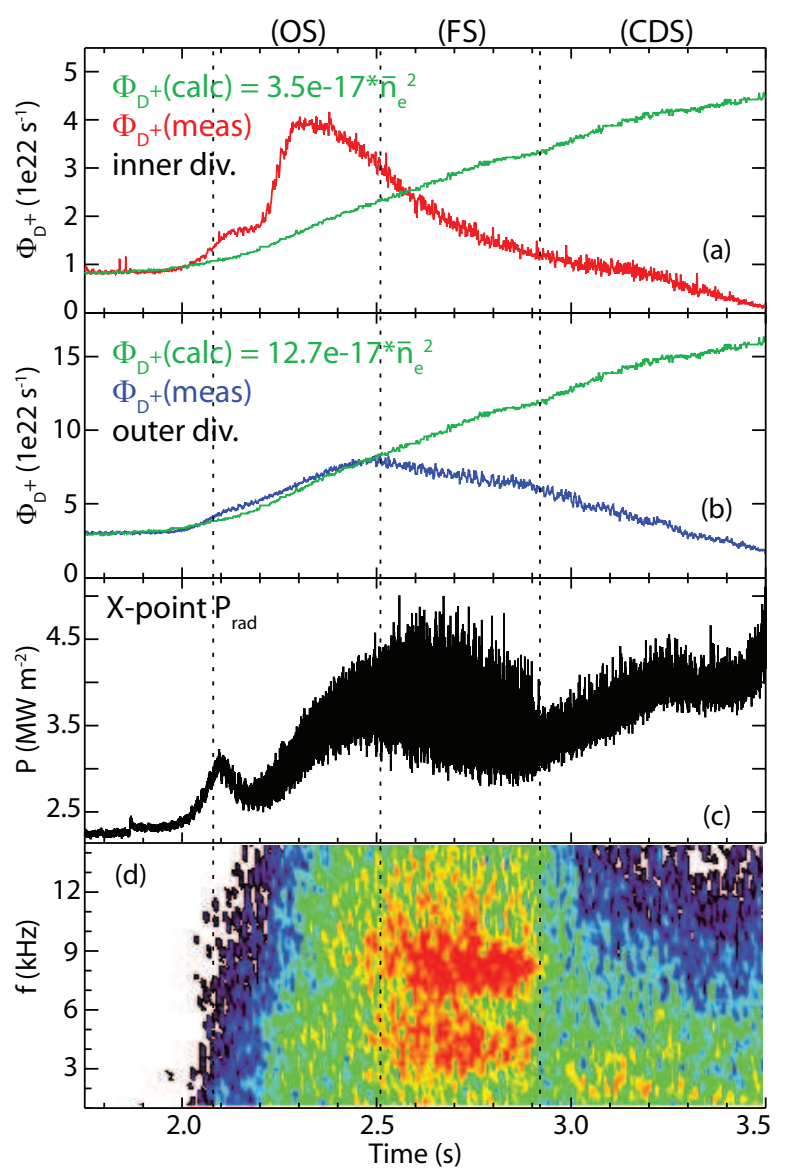

Figure 16. Calculated and measured total ion flux to the inner (a) and outer target (b), line integrated radiated power (c) measured by an AXUV diode (orange chord in Figure 2b) and its power spectrum (d) of discharge \# 27284. The three detachment states (OS), (FS) and (CDS) are indicated.

to $\approx 5 \cdot 10^{19} \mathrm{~m}^{-3}$. Similar to the inner divertor in forward field, the electron temperature at the target increases with increasing $\Gamma_{D^{+}}$and $n_{e, t}$ throughout this state (Fig. $17 \mathrm{~g}$ ). The density in the volume close to the strike point increases up to $\approx 1.5 \cdot 10^{20} \mathrm{~m}^{-3}$, see Figure 17c.

3.3.2. The fluctuating detachment state In reversed field, the start of this state is defined when the strength of the X-point fluctuations reaches its maximum, see Figure $16 \mathrm{~d}$.

The X-point fluctuations Contrary to forward field, two frequency bands, one at $f \approx 4.5 \mathrm{kHz}$ and one at $f \approx 9 \mathrm{kHz}$ are observed (Fig. 16d). The width of each of these frequency bands is $\Delta f \approx 3 \mathrm{kHz}$, which is similar to forward field.

The maximum of the fluctuations is still located in the inner SOL close to the Xpoint, see figure 18. Their spatial extent, however, is larger than in forward field and seems to expand into the outer divertor SOL. 


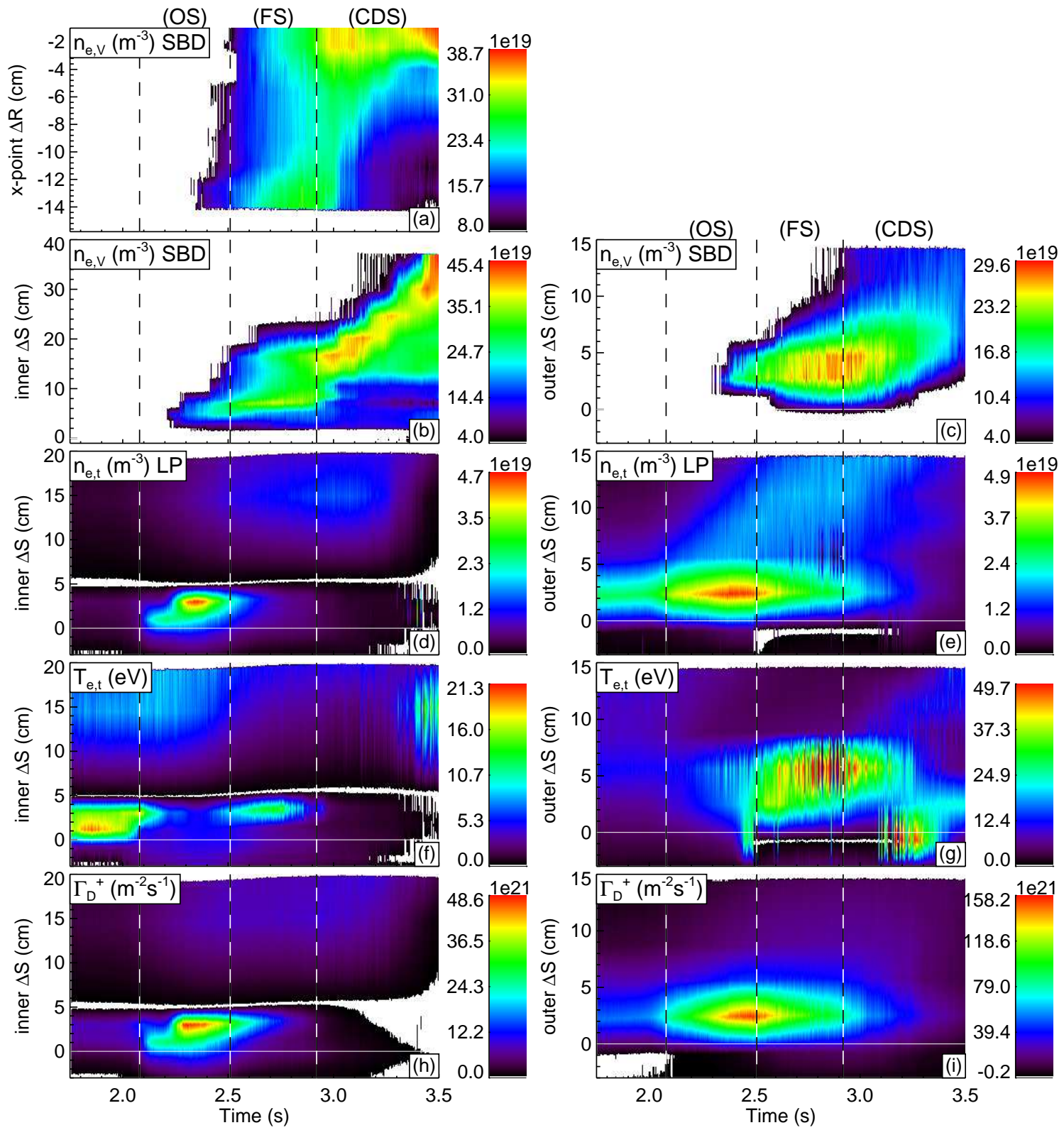

Figure 17. Horizontal (a) and vertical (b,c) $n_{e, V}$ profile in the divertor volume and $n_{e, t}(\mathrm{~d}, \mathrm{e}), T_{e, t}(\mathrm{f}, \mathrm{g})$ and $\Gamma_{D^{+}}(\mathrm{h}, \mathrm{i})$ target profiles in the inner and outer divertor, respectively, of discharge \# 27284. The three detachment states (OS), (FS) and (CDS) are indicated.

Inner divertor During this state, the ion flux and the target density steadily drop further to $\Gamma_{D^{+}} \approx 5 \cdot 10^{21} \mathrm{~m}^{-2} \mathrm{~s}^{-1}$ and $n_{e, t} \approx 7 \cdot 10^{18} \mathrm{~m}^{-3}$, respectively (Fig. $17 \mathrm{~d}, \mathrm{~h}$ ). Furthermore, the profile of $n_{e, t}$ is getting broader. The target temperature first increases further up to $T_{e, t} \approx 13 \mathrm{eV}$ and then rolls over within this state (Fig. 17f). Associated with the further decrease of $\Gamma_{D^{+}}$and $n_{e, t}$, the high density front at the strike point region $(\Delta S<10 \mathrm{~cm})$ rises up to $n_{e, V} \approx 3.7 \cdot 10^{20} \mathrm{~m}^{-3}$ and then rolls over when $T_{e, t}$ rolls over (Fig. 17b). Linked to the fluctuations high density fronts of $n_{e} \approx 3 \cdot 10^{20} \mathrm{~m}^{-3}$ (Fig 


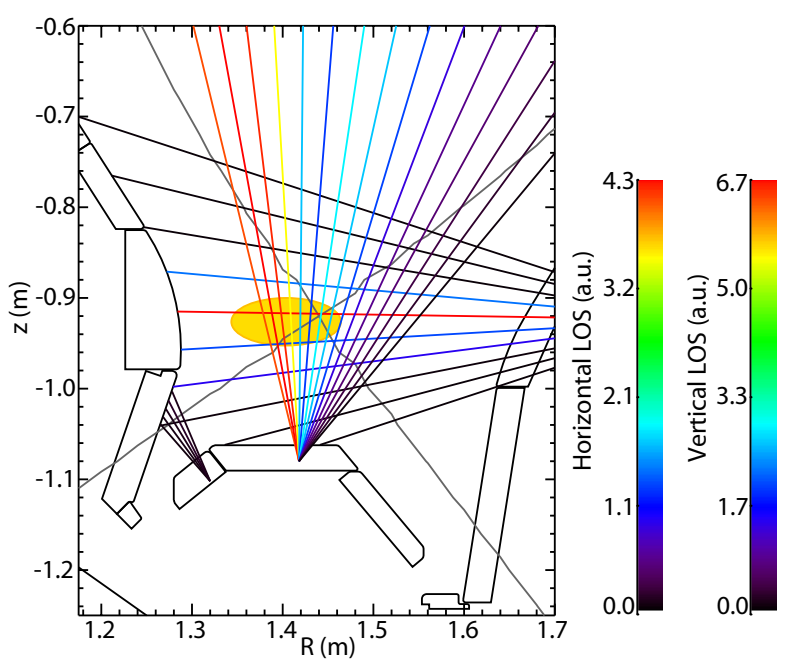

Figure 18. Intensity of the fluctuations for all AXUV channels of discharge \# 27284. The color-code represents the strength.

17a,b) develop in the inner far SOL $(\Delta S \approx 15 \mathrm{~cm})$ and X-point region $(\Delta R \approx-2 \mathrm{~cm})$. This is similar to forward field. Contrary to forward field, the X-point high density front reaches its maximum at the end of the fluctuating state.

Outer divertor $\Phi_{D^{+}}, \Gamma_{D^{+}}$and $n_{e, t}$ roll over at the start of this state (Figs. 16b, 17e,i) and the DOD exceeds unity (Fig. 15b). The target temperature steadily increases throughout this state up to $T_{e, t} \approx 50 \mathrm{eV}$ (Fig. $17 \mathrm{~g}$ ). Furthermore $T_{e, t}$ strongly fluctuates, which is most likely linked to the radiative $\mathrm{X}$-point fluctuations. As the time resolution of the Langmuir probes is too slow, a correlation analysis with the AXUV diodes can not, however, be made. Associated with the further decrease of $\Gamma_{D^{+}}$and $n_{e, t}$, the high density front at the strike point region increases up to $n_{e} \approx 3 \cdot 10^{20} \mathrm{~m}^{-3}$ during this state (Fig. 17c).

3.3.3. The complete detachment state The beginning of the complete detachment state is defined, similar to forward field, when the radiative $\mathrm{X}$-point fluctuations vanish. The finally achieved DOD is $\approx 40$ and $\approx 9$ for the inner and outer divertor, respectively (Fig. 15). Compared to the forward field case, this is much lower (inner DOD $\approx 600$, outer DOD $\approx 12$ ) but again higher in the inner divertor.

Inner divertor At the start of this state, the target parameters at the strike point region $(\Delta S<10 \mathrm{~cm})$ have already reached very low values of $\Gamma_{D^{+}}<2 \cdot 10^{21} \mathrm{~m}^{-2} \mathrm{~s}^{-1}$, $n_{e, t}<3 \cdot 10^{18} \mathrm{~m}^{-3}$ and $T_{e, t}<2 \mathrm{eV}$ (Fig. $17 \mathrm{~d}, \mathrm{f}, \mathrm{h}$ ). Also the high density front in this region has vanished (Fig. 17b) which, according to our definition, indicates that the plasma is completely detached from the inner strike point region. Moreover, similar to forward field, the high density front from the inner strike point region moves upwards along the field lines well above the X-point (Fig. 17a,b). 

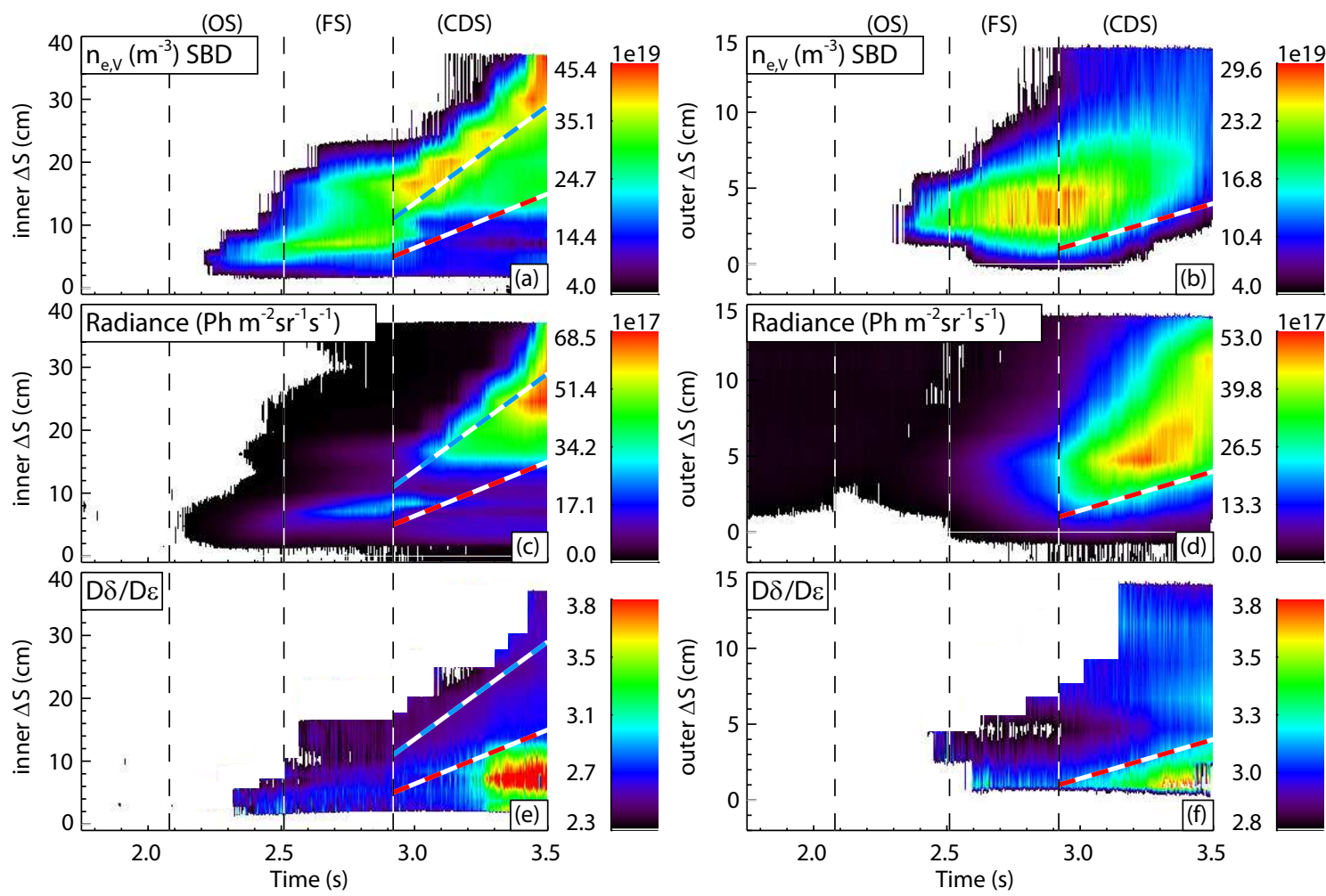

Figure 19. Profiles of $n_{e, V}(\mathrm{a}, \mathrm{b})$, the $\mathrm{D}_{\delta}$ emissivity $(\mathrm{c}, \mathrm{d})$ and the line ratio $\mathrm{D}_{\delta} / \mathrm{D}_{\epsilon}(\mathrm{e}, \mathrm{f})$ in the inner and outer divertor volume, respectively of discharge \# 27284. The three detachment states (OS), (FS) and (CDS) are indicated. See text for further explanation.

Outer divertor The ion flux and the density at the target drop further during this state (Fig. 17e,i). Similar to the inner divertor in forward field, the electron temperature at the strike point region $(\Delta S \approx 5 \mathrm{~cm})$ starts to decrease (here to $T_{e, t} \approx 6 \mathrm{eV}$ at the end of the discharge) while there is a short strong increase of $T_{e, t}$ in the private flux region (Fig. 17g). The high density front in the strike point region $(\Delta S<10 \mathrm{~cm}$, Fig. 17c) is reduced by $\approx 50 \%$ to $n_{e} \approx 1.5 \cdot 10^{20} \mathrm{~m}^{-3}$ during this state. The position of the peak $n_{e, V}$ moves slightly upstream, but, contrary to forward field, stays in the target region $(\Delta S<10 \mathrm{~cm})$ until almost the end of the discharge.

Evidence for volume recombination and low divertor temperatures Similar to forward field, there is evidence for volume recombination and thus electron temperatures below $\approx 1 \mathrm{eV}$ in the inner and outer divertor. Figure 19 shows the density in the divertor volume, the emissivity of $\mathrm{D}_{\delta}$ and the line ratio $\mathrm{D}_{\delta} / \mathrm{D}_{\epsilon}$. It can be seen again that the line emission increases when the high density front has moved away from the target (below the blue dashed line in Fig. 19a,c), indicating temperatures of $\approx 1.5 \mathrm{eV}$ when the emission reaches its maximum. When the line emission decreases, the $\mathrm{D}_{\delta} / \mathrm{D}_{\epsilon}$ line ratio increases, and electron temperatures below $\approx 1 \mathrm{eV}$ can be expected at times and regions below the red dashed lines in Figure 19. 


\subsection{Effect of magnetic perturbation fields}

When theses experiments were carried out, ASDEX Upgrade was equipped with eight in-vessel saddle coils [35] in order to externally produce magnetic perturbation fields (MP). They are located at the low field side, four coils are mounted above and four coils are mounted below the midplane. The discharge \#27101 was repeated with the coils switched on (Table 1). The magnetic perturbation field produced by the coils has a toroidal mode number of $n=2$ and the currents of the upper and lower coils, $I_{\text {coil }}=1 \mathrm{kA}$, are applied with odd parity.
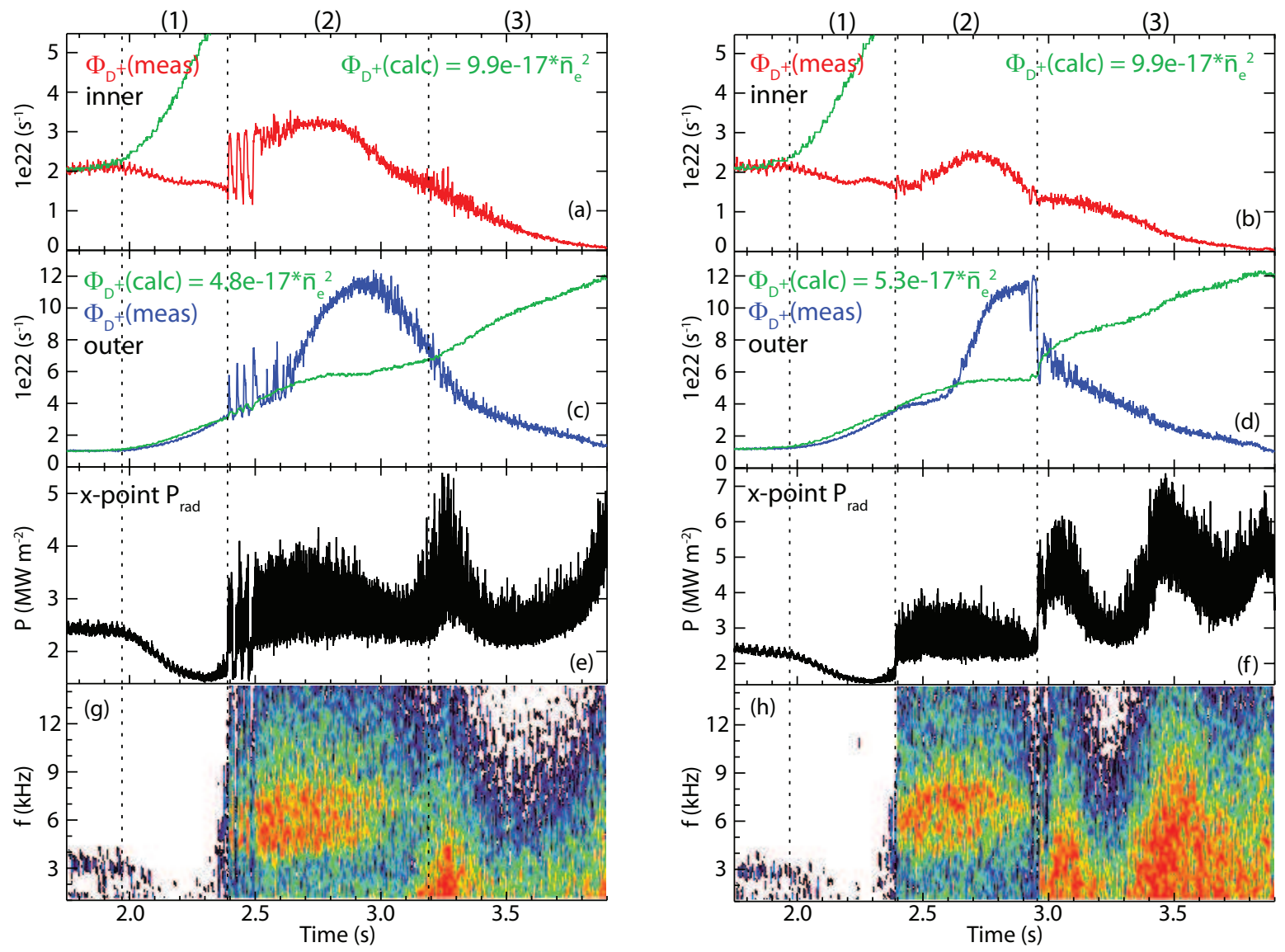

Figure 20. Calculated and measured total ion flux to the inner $(a, b)$ and outer target $(\mathrm{c}, \mathrm{d})$, line integrated radiated power $(\mathrm{e}, \mathrm{f})$ measured by an AXUV diode (orange chord in Figure $2 \mathrm{~b}$ ) and its power spectrum $(\mathrm{g}, \mathrm{h})$ without (left column, \# 27101) and with (right column, \# 27102) MP, respectively. The three detachment states (OS), (FS) and (CDS) are indicated.

Figure 20 shows $\Phi_{D^{+}}$and the power spectrum of an AXUV diode of discharge \# 27101 (without MP) in comparison with discharge \# 27102 (with MP). Before the onset of detachment, the total ion flux to the inner target is $2 \cdot 10^{22} \mathrm{~s}^{-1}$ with and without MP. With MP $\Phi_{D^{+}}$in the outer divertor is slightly higher $\left(\Phi_{D^{+}}=1.8 \cdot 10^{22} \mathrm{~s}^{-1}\right)$ compared to the case without MP $\left(\Phi_{D^{+}}=1.6 \cdot 10^{22} \mathrm{~s}^{-1}\right)$. The onset of detachment and the transition into the fluctuating state occur with and without MP at the same time. 

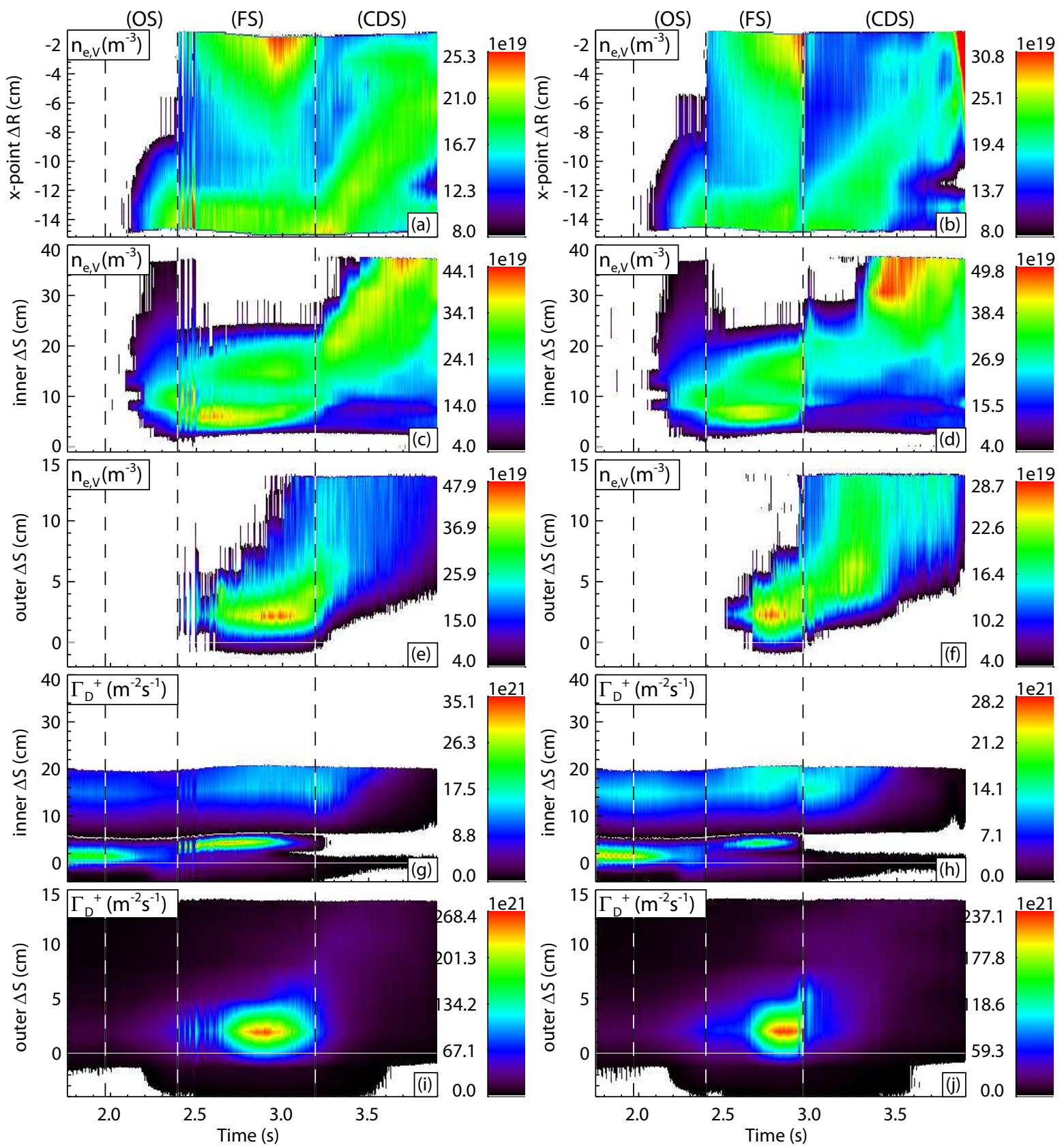

Figure 21. Horizontal (a,b) and vertical $(\mathrm{c}, \mathrm{d}) n_{e, V}$ and $\Gamma_{D^{+}}(\mathrm{g}, \mathrm{h})$ profiles in the inner divertor. Vertical $(\mathrm{e}, \mathrm{f}) n_{e, V}$ and $\Gamma_{D^{+}}(\mathrm{i}, \mathrm{j})$ profiles in the outer divertor. Left column without MP (\#27101), right column with MP (\#27102). The three detachment states (OS), (FS) and (CDS) are indicated. 
Also the temporal evolution of $\Gamma_{D^{+}}$and $n_{e, V}$, which are shown in Figure 21 for both discharges, evolve with and without MP similar up to the fluctuating state. The peaks of $\Phi_{D^{+}}$at the beginning of the fluctuating state without MP (Fig. 20a,c $t=2.4 \mathrm{~s}$ ) are the so called divertor plasma oscillations which are discussed in [34] and are not of importance for this comparison.

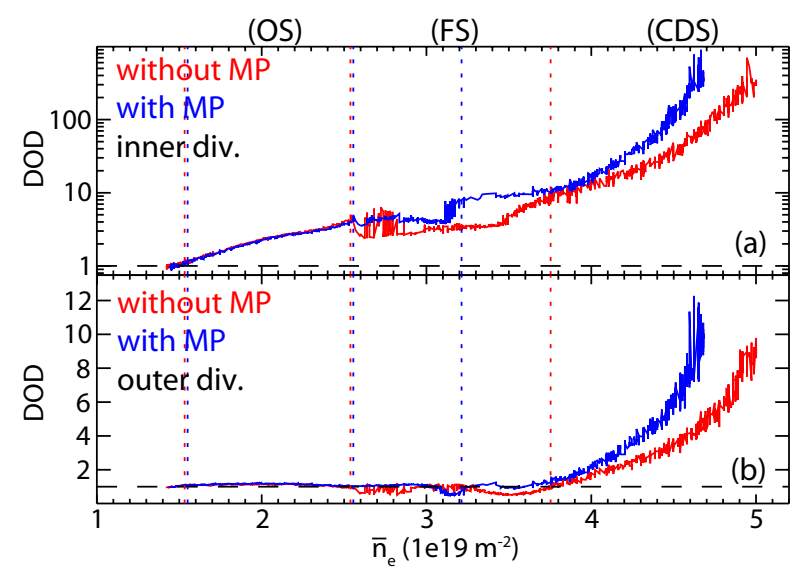

Figure 22. DOD as a function of the line integrated edge plasma density for the inner (a) and outer (b) divertor without (red, \# 27101) and with (blue, \# 27102) MP. Note the logarithmic scale of (a). The three detachment states (OS), (FS) and (CDS) are indicated.

The main difference in the evolution of divertor detachment with and without MP begins when the outer divertor starts to detach, i.e. when the outer $\Gamma_{D^{+}}$rolls over. With MP the outer $\Gamma_{D^{+}}$then drops abruptly (Fig. 20d) and the X-point fluctuations vanish (Fig. 20h), which determines the end of the fluctuating state. In the case without MP, there is a smooth roll over of the outer $\Gamma_{D^{+}}$(Fig. 20c) and the end of the fluctuating state (Fig. 20g) happens later than in the MP case.

The complete detachment state with MP can be divided into two parts. At the beginning of the complete detachment state the high density fronts in the inner far SOL and X-point region disappear (Fig. 21b,d) as abruptly as the drop of the ion flux to the outer target. At the same time there is a sharp strong increase of the main plasma density (see $\Phi_{D^{+}}^{\text {calc }}$ in Figure $20 \mathrm{~d}$ which is proportional to the main plasma density). The plasma is completely detached from the inner strike point region $\left(\Gamma_{D^{+}}(\Delta S<8 \mathrm{~cm})<1 \cdot 10^{21} \mathrm{~m}^{-2} \mathrm{~s}^{-1}\right)$ at the beginning of the CDS, but the peak $\Gamma_{D^{+}}$in the far SOL shifts towards the strike point (Fig. $21 \mathrm{~h}$ ). The peak $\Gamma_{D^{+}}$at the outer strike point drops at the beginning of the complete detachment state to $\Gamma_{D^{+}} \approx 6.9 \cdot 10^{22} \mathrm{~m}^{-2} \mathrm{~s}^{-1}$, which is comparable to the case without MP (Fig. 21i,j). The high density front in the outer divertor moves upstream $(\Delta S=2 \mathrm{~cm}$ to $\Delta S=5 \mathrm{~cm})$ and, contrary to the case without MP, stays in this region (compare Fig. 21e,f, $\geq 3.2 \mathrm{~s}$ ). During the second part of the complete detachment state with MP this outer high density front moves out of the region covered by the Stark diagnostic. The DOD with and without MP is shown in Figure 22. It can be seen that the complete detachment state starts slightly earlier with 
$\operatorname{MP}\left(\bar{n}_{e}=3.2 \cdot 10^{19} \mathrm{~m}^{-2}\right.$ without MP and $\bar{n}_{e}=3.8 \cdot 10^{19} \mathrm{~m}^{-2}$ with MP). Furthermore, with MP detachment in the inner and outer divertor proceeds faster with respect to the upstream density. Consequently, the density limit occurs earlier with MP, i.e. at $\bar{n}_{e}=4.6 \cdot 10^{19} \mathrm{~m}^{-2}$ compared to $\bar{n}_{e}=5 \cdot 10^{19} \mathrm{~m}^{-2}$ without MP.

\section{Discussion}

Role of $\vec{E} \times \vec{B}$ drifts It was proposed in Ref. [15] that plasma flows induced by a combination of radial and poloidal $\vec{E} \times \vec{B}$ drifts, which change direction when the field is changed [36], drive asymmetries of the ion flux reaching the inner and outer divertor. Due to the variation of the pre-sheath potential drop a poloidal electric field $E_{\Theta}$ is formed in front of the target. The resulting $E_{\Theta} \times B$ drift induces a radial flow $\Gamma_{r}^{d r}$. In forward field this flow is directed from the outer SOL across the separatrix into the private flux region $(\mathrm{PF})$ and from the $\mathrm{PF}$ into the inner SOL. In addition, a radial electric field $E_{r}$ in the PF results in a poloidal flow $\Gamma_{\Theta}^{d r}$ induced by the $E_{r} \times B$ drift. This flow in the $\mathrm{PF}$ is in forward field directed from the outer to the inner divertor. For similar conditions, such a flow in the PF has been measured in JT-60U [37] and ASDEX Upgrade [38]. Thus, the combination of both, the $\Gamma_{\Theta}^{d r}$ flow in the PF and the $\Gamma_{r}^{d r}$ flow brings more plasma to the inner divertor in forward field and to the outer divertor in reversed field. It was further shown at ASDEX Upgrade that the $\vec{E} \times \vec{B}$ drifts have to be taken into account in order to simulate low density attached divertor plasmas correctly [39].

Such an asymmetry was also observed here. At the lowest density before the fueling ramp starts, the ratio of the total ion flux reaching the inner and outer divertor in forward field is asymmetric and in favor of the inner divertor $\left(\Phi_{D^{+}}^{\text {in }} / \Phi_{D^{+}}^{\text {out }}=1.9\right)$. In reversed field, this ratio is still asymmetric but now being in favor of the outer divertor $\left(\Phi_{D^{+}}^{\text {in }} / \Phi_{D^{+}}^{\text {out }}=0.3\right)$. It was proposed, however, that the influence of these $\vec{E} \times \vec{B}$ drifts becomes less important at high densities due to the flatter temperature gradients [36]. Indeed, when the density is increased here, the roll over of $\Phi_{D^{+}}$happens first in the inner divertor for both field directions. The ratio of the edge line integrated plasma density at the inner and outer roll over is in reversed field $\bar{n}_{e, \text { in }} / \bar{n}_{e, \text { out }} \approx 2.1 / 2.5=0.84$. In forward field, this ratio is $\bar{n}_{e, \text { in }} / \bar{n}_{e, \text { out }} \approx 1.5 / 3.3=0.76$. Taking the second roll over (see next point) in forward field, the ratio is $\bar{n}_{e, \text { in }} / \bar{n}_{e, \text { out }} \approx 2.9 / 3.3=0.88$, which is even more comparable to the reversed field case. In addition, the finally achieved DOD is for both field directions larger in the inner divertor. Also the X-point fluctuations and the associated high density front in the far SOL occur for both field direction in the inner divertor. This shows that the $\vec{E} \times \vec{B}$ drifts contribute at low density but they have a minor influence on the overall detachment process.

Plasma evolution during the fluctuating detachment state At the beginning of the fluctuating detachment state the total ion flux, $\Phi_{D^{+}}$, to the inner divertor and $\Gamma_{D^{+}}$ and $n_{e, t}$ close to the inner strike point suddenly increase. In addition, there is a jump of 
the peak $\Gamma_{D^{+}}$and $n_{e, t}$ positions in the strike point region from $\Delta S \approx 1 \mathrm{~cm}$ to $\Delta S \approx 3 \mathrm{~cm}$ at the transition to this state. With increasing upstream density, $\Gamma_{D^{+}}$and $n_{e, t}$ at the strike point region $(\Delta S \approx 3 \mathrm{~cm})$ roll over the second time (which will be discussed later). After their roll over, $T_{e, t}$ increases in this narrow region at $\Delta S \approx 3 \mathrm{~cm}$ while the pressure remains approximately constant. CX-collisions are thus most likely not the cause of the ion flux reduction in the $\Delta S \approx 3 \mathrm{~cm}$ region (via $f_{\text {mom }}>0$ in eq. 5). Moreover, they are only effective at temperatures below $\approx 5 \mathrm{eV}[8]$, which is not the case in this region. An increase of the power being convected $\left(f_{\text {conv }}>0\right)$ leads also to a reduction of $\Gamma_{D^{+}}$ (eq. 5) and $n_{e, t}$ (eq. 4) and to an increase of $T_{e, t}$ (eq. 3), being in agreement with the measured evolution. Such a change of the ratio of conducted and convected power has been measured in the outer divertor of DIII-D [40]. Furthermore, in order to increase the fraction of convected power, the ionization front must move or expand into the SOL, which is consistent with the measured high density front in the inner divertor volume. The position and spatial extend of this high density front can be correlated with the total radiation distribution in the divertor (red circle in Fig. 23a) as recombination processes can be neglected at this point.

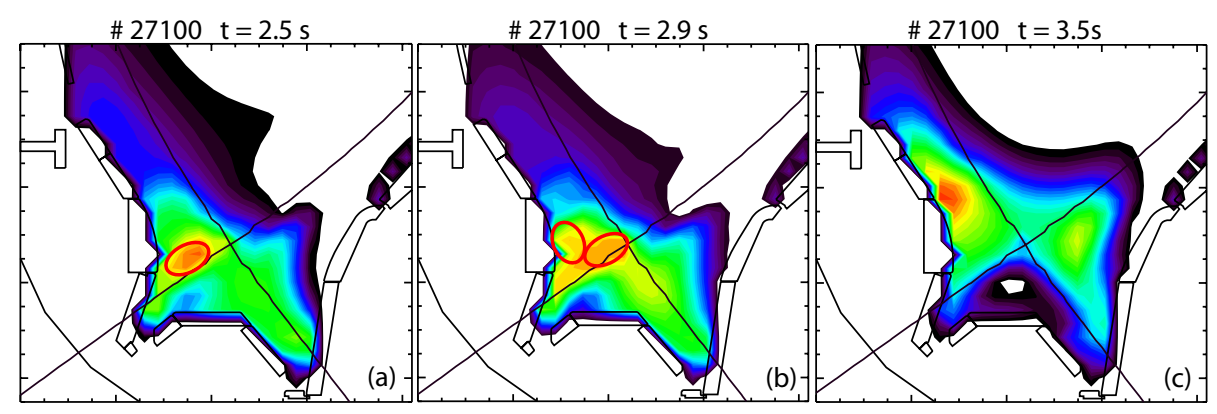

Figure 23. Total radiation distribution from foil bolometry in the divertor for three different time points of discharge \# 27100. See text for explanations.

At the end of the fluctuating state, the fueling of the main plasma becomes less efficient, i.e. although the fueling puff is constantly increased, the main plasma density almost saturates. Based on Krasheninnikov's stability theory of detached plasmas [41], the main plasma density cannot be increased further by gas puffing at a certain point. Then, the cold and dense plasma buffer, which is situated between the recombination and ionization front, must increase and move towards the X-point. Indeed, a decrease of $n_{e, V}$ at the inner strike point region and an increase of $n_{e, V}$ in the inner far SOL and $\mathrm{X}$-point region is measured during the phase in which the main plasma density increases less strongly than the fueling puff (compare also Fig.23a,b).

The two roll overs in forward field In forward field, two roll overs of $\Gamma_{D^{+}}$are observed in the inner divertor strike point region, one at the onset state $(\Delta S \approx 1 \mathrm{~cm})$ and one during the fluctuating detachment state $(\Delta S \approx 3 \mathrm{~cm})$ while there is no second roll over in the outer divertor, neither in forward nor in reversed field. 


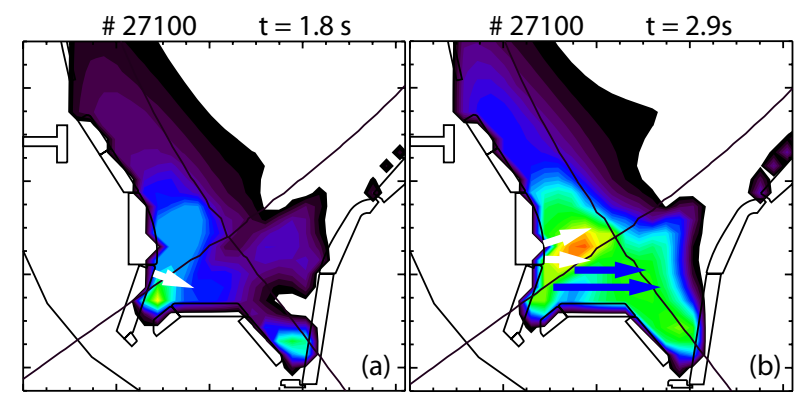

Figure 24. Total radiation distribution in the divertor for two different time points of discharge \# 27100. See text for explanations.

A possible reason for the early first roll over followed by a second roll over in the inner divertor in forward field could be a geometric effect of the divertor structure. The lower tile of the inner divertor target is tilted towards the separatrix, i.e. the angle between the target normal and the horizontal axis is negative (white arrow in Fig. 24a). During the onset state the ion flux profile is radially not very broad, which is supported by the total radiation distribution (Fig. 24a). Neutrals are recycled from the lower inner target and released in a $\cos ^{2}$ distribution cone around the target normal, hence back to the strike point region (white arrow in Fig. 24a). This would enhance the neutral pressure in the inner strike point region, which increases the probability for CX collisions to remove momentum, thus the ion flux decreases if not compensated by an increase of the power loss (eq. 5). This increase of the ion flux was observed. At the transition to the fluctuating state, a mechanism sets in which brings more particles to the far SOL, i.e. to the upper tile of the inner target (Fig. 24b). Once the ion fluxes to this upper tile are strong, and therefore recycling from this tile becomes strong, the situation should change. The upper tile curves backwards, i.e. the angle between the target normal and the horizontal axis becomes positive. When recycling takes place at this upper tile (high ion fluxes are measured in this region during the fluctuating state, confirmed by the radiation distribution Fig. 24b), neutrals will be released into regions further upstream (white arrows in Fig. 24b). This should change the divertor plasma conditions and could be the reason for the second increase and roll over of the ion flux in the inner divertor. In order to verify this theory, similar discharges can be made with different strike point positions. With a lower strike point, a higher density should be required to push the ion flux to the upper tile of the inner divertor.

The outer target, however, is constantly tilted such that the recycled neutrals will be released back to the separatrix leg. In reversed field, $\Phi_{D^{+}}$in the outer divertor\| scales longer with the TPM compared to forward field, then rolls over and decreases continuously. No second roll over is observed, which is consistent with this divertor geometry model.

\| As in forward field the inner divertor receives the higher ion flux before the onset of detachment while in reversed field this is the outer divertor, these two cases are compared 
Flux enhancement The flux enhancement, which is defined here when the total ion flux exceeds the Two-Point Model scaling, occurs in the outer divertor in forward field and in the inner divertor in reversed field. In forward field $\uparrow$, during the fluctuating state, a high electron density is measured in the inner far SOL and X-point region, while the density in the strike point region is reduced (see the radiation distribution $24 \mathrm{~b}$ as an indication). Namely, the inner divertor strike point region becomes transparent for neutrals (blue arrows in Fig. 24b). Recycled neutrals, originating from the strike point region, could therefore pass the inner SOL and the private flux region and reach the outer SOL. It has been shown previously at ASDEX Upgrade [42] that, under similar conditions, the inner divertor becomes transparent for neutrals and the neutral fluxes measured in the inner divertor and private flux region are equal. These neutrals, which reach the outer divertor SOL, will ionize there. This leads to additional radiation losses $\left(f_{\text {pow }}>0\right)$ in the outer SOL. As a consequence, the ion flux at the target increases (eq. $5)$, consistent with the observations. In addition, these ionized neutrals should provide an additional particle source in the outer SOL, resulting in a fraction of power being convected $\left(f_{\text {conv }}>0\right)$. This may explain the increase of the outer target temperature (eq. 3) during the flux enhancement phase, while the target ion flux and density also increase. In total, this would then be a combination, or competition, of the $f_{\text {pow }}>0$ and $f_{\text {conv }}>0$ effects. The flux enhancement might be similar to the death rays observed in Alcator C-Mod [13] where the plasma pressure at the target sometimes exceeds the upstream values prior to detachment. But this effect was localized in narrow region close to the strike point while the flux enhancement, observed here, occurs over a large target area and during the detachment process in every discharge.

Radiative $X$-point fluctuations Once the X-point fluctuations are triggered, a high electron density and Balmer radiance is always measured at the $\mathrm{X}$-point region. This indicates that the ionization front expands up the X-point, which may cause this phenomena, pointing towards a MARFE like instability. The frequency of the fluctuations depends inversely on the square root of the mass of the fueling species. It was further shown in Ref. [34] that the injection of additional nitrogen also decreases the frequency. This points in the same direction, as with nitrogen the effective charge of the plasma, $Z_{\text {eff }}$, is increased.

This mass dependence on the frequency suggests that the ion sound speed, $c_{s}=$ $\sqrt{k_{B}\left(T_{e}+T_{i}\right) / m_{i}}$, is somehow involved. E.g. ions/filaments, originating at the X-point due to the ionization of recycled neutrals (MARFE like), flow with $c_{s}$ from the Xpoint to the inner or outer target. This would be consistent with the increased fraction of convected power $\left(f_{\text {conv }}>0\right)$ in the $\Delta S \approx 3 \mathrm{~cm}$ region of the inner divertor, as discussed above. An assumption of $T_{i}=T_{e}=15 \mathrm{eV}$, which is reasonable for ionizing conditions, gives $c_{s} \approx 38 \mathrm{~km} / \mathrm{s}$. The connection length from the inner X-point, starting at a horizontal distance from the $\mathrm{X}$-point which corresponds to $\Delta S \approx 3 \mathrm{~cm}$ at the target

ๆ In the explanation for reversed field, the inner and outer divertor would simply change 
$\left(\approx 7 \mathrm{~cm}\right.$ in real space or $\rho_{\text {pol }} \approx 1.004$ in normalized flux $)$ towards the outer divertor is $L_{c} \approx 62 \mathrm{~m}$. This yields a characteristic frequency of $f \approx 0.6 \mathrm{kHz}$ which is too low. The connection length to the inner divertor, $L_{c} \approx 6.4 \mathrm{~m}$ ), gives a frequency of $f \approx 5.9 \mathrm{kHz}$. This is a reasonable agreement, despite the uncertainty in the calculation of $c_{s}$ and $L_{c}$. The underlying physical mechanism, however, cannot be explained here. As a first step, an additional perpendicular transport in the inner X-point region was incorporated into numerical simulations [43]. It should also be mentioned that this instability may also be a kind of limit cycle. The ionization front close to X-point leads to an instability which transports the plasma away, e.g. in the far SOL. The inner divertor is then shortly re-attached and starts to detach again. It cannot be verified, however, whether the density oscillates with the frequency of the fluctuations between the high density front at the X-point and the far SOL or strike point region, as the time resolution of the Stark broadening diagnostic and the Langmuir probes is too slow. New and faster diagnostics are therefore needed to gain more information on this topic.

\section{Summary and Conclusion}

The objective of this paper was to present an experimental investigation of the evolution of divertor detachment at ASDEX Upgrade (AUG). With new diagnostics a better knowledge of the plasma parameters, especially of the electron density in the divertor volume, was obtained, resulting in a consistent picture of the detachment process.

The main result of this work is a new classification of divertor detachment. The detachment process is not continuously evolving but undergoes three different states where the inner and outer divertor plasma conditions are strongly coupled. It starts with the onset state, when the first deviation of the target ion flux from the simple Two-Point Model (TPM) occurs. This happens in the inner divertor with the roll over of the ion flux (DOD > 1) close to the strike point, while the outer divertor remains attached during this state $(\mathrm{DOD}=1)$.

The appearance of radiative fluctuations, which are observed for the first time, characterizes the start of the fluctuating detachment state. These fluctuations have a mean frequency of $f \approx 5.5 \mathrm{kHz}$, a width of $\Delta f \approx 3 \mathrm{kHz}$ and are situated in the inner SOL close to the X-point. From deuterium to hydrogen, the mean frequency is increased by the square root of the mass ratio of both species, i.e. $f \approx 8 \mathrm{kHz}$. In addition, regions of densities, one order of magnitude higher than the main plasma density, develop in the inner far SOL and X-point regions. The outer divertor is still attached during this state, but the ion flux exceeds the TPM scaling (DOD $<1$ ). This so-called flux enhancement is most likely caused by an enhanced ionization of recycled neutrals originating from the inner divertor. As the inner divertor is already partially detached, it becomes transparent for recycled neutrals, which can now reach the outer SOL where they are ionized. The inner divertor plasma thus influences the outer one and encourages the outer divertor detachment. In conclusion, in order to be able to model the outer divertor detachment at AUG correctly, one must be able to reproduce the 
detached plasma conditions in the inner divertor together with the X-point fluctuations.

The transition to the complete detachment state happens when the radiative Xpoint fluctuations vanish. At this point the inner and outer divertor simultaneously start to detach completely from the strike point regions. The complete detachment is defined here when the target parameters $\Gamma_{D^{+}}, n_{e, t}$ and $T_{e, t}$ drop to significantly lower values and, the main point, when the high density fronts in the strike point regions vanish. During the complete detachment state, the high density fronts move from the targets towards and even above the X-point, resulting in a MARFE and a density limit disruption. It was shown by means of spectroscopy, that, once the high density fronts have moved away from the targets, recombination is dominant and temperatures are below $\approx 1 \mathrm{eV}$ only during this state and in the regions between the high density fronts and the targets.

The three detachment states are observed in reversed field, too. The radiative $\mathrm{X}$ point fluctuations now consist of two frequency bands of $f \approx 4.5 \mathrm{kHz}$ and $f \approx 9 \mathrm{kHz}$, but they are again located in the inner SOL close to the X-point. Moreover, the $\vec{E} \times \vec{B}$ drifts were found to contribute at low density but they have a minor influence on the overall detachment process.

Finally, the effect of an additional magnetic perturbation field (MP) on the detachment process was studied. Detachment proceeds similarly with and without MP until the ion flux to the outer divertor rolls over. With MP, this roll over is followed by an abrupt and strong drop of the ion flux in the outer divertor and the electron density in the inner far SOL and X-point region, associated with an abrupt strong increase of the main plasma density. Moreover, detachment starts slightly earlier and proceeds faster with MP.

\section{References}

[1] R.A. Pitts, S. Carpentier, F. Escourbiac, T. Hirai, V. Komarov, S. Lisgo, A.S. Kukushkin, A. Loarte, M. Merola, A. Sashala Naik, R. Mitteau, M. Sugihara, B. Bazylev, and P.C. Stangeby. A full tungsten divertor for ITER: Physics issues and design status. J. Nucl. Mater., 438:48-56, 2013.

[2] A.S. Kukushkin, H.D. Pacher, G.W. Pacher, G. Janeschitz, D. Coster, A. Loarte, and D. Reiter. Scaling laws for edge plasma parameters in ITER from two-dimensional edge modelling. Nucl. Fusion, 43:716, 2003.

[3] S. I. Krasheninnikov. Two-dimensional effects in plasma radiation fronts and radiation front jumps in tokamak divertor plasmas. Phys. Plasmas, 4(11):3741-3743, 1997.

[4] S.I. Krasheninnikov, M. Rensink, T.D. Rognlien, A.S. Kukushkin, J.A. Goetz, B. LaBombard, B. Lipschultz, J.L. Terry, and M. Umansky. Stability of the detachment front in a tokamak divertor. J. Nucl. Mater., 266269:251-257, 1999.

[5] P.C. Stangeby. Can detached divertor plasmas be explained as self-sustained gas targets? Nucl. Fusion, 33:1695, 1993.

[6] C.S. Pitcher and P.C. Stangeby. Experimental divertor physics. Plasma Phys. Control. Fusion, 39:779-930, 1997.

[7] R. Schneider, X. Bonnin, K. Borras, D. Coster, H. Kastelewicz, D. Reiter, V.A. Rozhansky, and J. Braams. Plasma edge physics with B2-Eirene. Contrib. Plasma Phys., 46:3-191, 2006. 
[8] B. Lipschultz, B. LaBombard, J.L. Terry, C. Boswell, and I.H. Hutchinson. Divertor physics research on Alcator C-Mod. Fusion Science And Technology, 51(3):369-389, 2007.

[9] M. Wischmeier, M. Groth, A. Kallenbach, A. V. Chankin, D. P. Coster, R. Dux, A. Herrmann, H. W. Müller, R. Pugno, D. Reiter, A. Scarabosio, J. G. Watkins, DIII-D team, and ASDEX Upgrade Team. Current understanding of divertor detachment: Experiments and modelling. J. Nucl. Mater., 390-391:250-254, 2009.

[10] A.W. Leonard, C.J. Lasnier, J.W. Cuthbertson, T.E. Evans, M.E. Fenstermacher, N. Hill, D, R.A. Jong, W.H. Meyer, T.W. Petrie, and G.D. Porter. Power balance in DIII-D during single-null ELMing H-mode plasmas. J. Nucl. Mater., 220-222:325-329, 1995.

[11] A.V. Chankin, D.J. Campell, S. Clement, S.J. Davies, L.D. Horton, J. Lingertat, A. Loarte, G.F. Matthews, R.D. Monk, R. Reichle, G. Saibene, M. Stamp, and P.C. Stangeby. Toroidal field reversal effects on divertor asymmetries in JET. Plasma Phys. Control. Fusion, 38:1579-1592, 1996.

[12] G.M. Staebler. The critical point for the onset of divertor energy flux asymmetry in tokamaks. Nucl. Fusion, 36:1437-1453, 1996.

[13] B. LaBombard, J.A. Goetz, and I. Hutchinson. Experimental investigation of transport phenomena in the scrape-off layer and divertor. J. Nucl. Mater., 241-243:149-166, 1997.

[14] M. Shimada and T. Ohkawa. Effect of gyro-motion of incident ions on inboard-outboard asymmetry in divertor plasmas. J. Nucl. Mater., 266-269:906-910, 1999.

[15] R.A. Pitts. Edge and divertor physics with reversed toroidal field in JET. J. Nucl. Mater., 337-339:146-153, 2005.

[16] A. Huber. The effect of field reversal on the JET MkIIGB-SRP divertor performance in L-mode density limit discharges. J. Nucl. Mater., 337-339:241-245, 2005.

[17] J.A. Boedo, D. Rudakov, and R. Moyer. Transport by intermittent convection in the boundary of the DIII-D tokamak. Phys. Plasmas, 8:4826-4834, 2001.

[18] J.P. Graves, J. Horacek, R.A. Pitts, and K.I. Hopcraft. Self-similar density turbulence in the TCV tokamak scrape-off layer. Plasma Phys. Control. Fusion, 47:L1-L9, 2005.

[19] S. Wiesen, W. Fundamenski, M. Wischmeier, M. Groth, S. Brezinsek, V. Naulin, and JET EFDA contributors. Relevance of collisionality in the transport model assumptions for divertor detachment multi-fluid modelling on JET. J. Nucl. Mater., 415:S535-S539, 2011.

[20] R. Neu, V. Bobkov, R. Dux, J.C. Fuchs, O. Gruber, A. Herrmann, A. Kallenbach, H. Maier, M. Mayer, T. Pütterich, V. Rohde, A.C.C. Sips, J. Stober, K. Sugiyama, and ASDEX Upgrade Team. Ten years of W programme in ASDEX Upgrade - challenges and conclusions. Phys. Scr., T138:014038 (6pp), 2009.

[21] A.G. Meigs, S. Brezinsek, M. Clever, A. Huber, S. Marsen, C. Nicholas, M. Stamp, K.D. Zastrow, and JET EFDA contributors. First results from mirror-link divertor spectroscopy system on the JET ITER-Like wall. J. Nucl. Mater., 438:S607-S611, 2013.

[22] J.R. Harrison, S.W. Lisgo, K.J. Gibson, P. Tamain, J. Dowling, and The MAST Team. Characterisation of detached plasmas on the MAST tokamak. J. Nucl. Mater., 415:S379-S382, 2011.

[23] C. Stehlé and R. Hutcheon. Extensive tabulations of Stark broadened hydrogen line profiles. Astron. Astrophys. SuppL. Ser., 140:93-97, 1999.

[24] S. Potzel, R. Dux, H.W. Müller, A. Scarabosio, M. Wischmeier, and ASDEX Upgrade Team. Electron density determination in the divertor volume of ASDEX Upgrade via Stark broadening of the Balmer lines. submitted to Plasma Phys. Control. Fusion.

[25] B. Reiter, G. Pautasso, T. Eich, J.C. Fuchs, L. Giannone, R. Dux, J. Neuhauser, M. Maraschek, V. Igochine, A. Herrmann, T. Lunt, and ASDEX Upgrade Team. Application of AXUV Diodes for broad-band plasma radiation studies in ASDEX Upgrade. In M Mateev and E Benova, editors, Europhysics Conference Abstracts (CD-ROM, Proc. of the 36th EPS Conference on Plasma Physics, Sofia, Bulgaria, 2009), volume 33E of ECA, pages P-1.161, Geneva, 2009. European Physical Society. 
[26] O. Schmitz, I.L. Beigman, L.A. Vainshtein, B. Schweer, M. Kantor, A. Pospieszczyk, Y. Xu, M. Krychowiak, M. Lehnen, U. Samm, B. Unterberg, and the TEXTOR team. Status of electron temperature and density measurement with beam emission spectroscopy on thermal helium at TEXTOR. Plasma Phys. Control. Fusion, 50(11):115004, 2008.

[27] K. Fujimoto, T. Nakano, H. Kubo, K. Sawada, T.Takizuka, H. Kawashima, K. Shimizu, and N. Asakura. Spatial structure of volume recombination in JT-60U detached divertor plasmas. Plasma Fusion Res., 4:025, 2009.

[28] A. Huber, S. Brezinsek, M. Groth, P.C. de Vries, V. Riccardo, G. van Rooij, G. Sergienko, G. Arnoux, A. Boboc, P. Bilkova, G. Calabro, M. Clever, J.W. Coenen, M.N.A. Beurskens, T. Eich, S. Jachmich, M. Lehnen, E. Lerche, S. Marsen, G.F. Matthews, K. McCormick, A.G. Meigs, Ph. Mertens, V. Philipps, J. Rapp, U. Samm, M. Stamp, M. Wischmeier, and S. Wiesen. Impact of the ITER-Like wall on divertor detachment and on the density limit in the JET tokamak. J. Nucl. Mater., 438:S139-S147, 2013.

[29] H.P. Summers, W.J. Dickson, M.G. O’Mullane, N.R. Badnell, A.D. Whiteford, D.H. Brooks, J. Lang, S.D. Loch, and D.C. Griffin. Ionization state, excited populations and emission of impurities in dynamic finite density plasmas: I. The generalized collisional-radiative model for light elements. Plasma Phys. Control. Fusion, 48(2):263, 2006.

[30] P.C. Stangeby. The Plasma Boundary of Magnetic Fusion Devices. Institute of Physics Publishing Bristol and Philadelphia, 2000.

[31] Keilhacker. Plasma Physics and Controlled Nuclear Fusion Research, III:183, 1982.

[32] A. Loarte. Plasma detachment in JET Mark I divertor experiments. Nucl. Fusion, 38:331, 1998.

[33] G.D. Porter, S. Davies, B. LaBombard, A. Loarte, K. McCormick, R. Monk, M. Shimada, and M. Sugihara. Analysis of separatrix plasma parameters using local and multi-machine databases. J. Nucl. Mater., 266-269:917-921, 1999.

[34] S. Potzel, M. Wischmeier, M. Bernert, R. Dux, H.W. Müller, A. Scarabosio, and ASDEX Upgrade Team. Characterization of the fluctuating detachment state in ASDEX Upgrade. J. Nucl. Mater., 438:285-290, 2013.

[35] W. Suttrop, O. Gruber, S. Günter, D. Hahn, A. Herrmann, M. Rott, T. Vierle, U. Seidel, M. Sempf, B. Streibl, E. Strumberger, D. Yadikin, O. Neubauer, B. Unterberg, E. Gaio, V. Toigo, P. Brunsell, and ASDEX Upgrade Team. In-vessel saddle coils for MHD control in ASDEX Upgrade. Fusion Eng. and Des., 84(2-6):290-294, 2009.

[36] P.C. Stangeby and A.V. Chankin. Simple models for the radial and poloidal $E \times B$ drifts in the scrape-off layer of a divertor tokamak: Effects on in/out asymmetries. Nucl. Fusion, 36:839-852, 1996.

[37] N. Asakura, H. Takenaga, S. Sakurai, G.D. Porter, T.D. Rognlien, M.E. Rensink, K. Shimizu, S. Higashijima, and H. Kubo. Driving mechanism of SOL plasma flow and effects on the divertor performance in JT-60U. Nucl. Fusion, 44:503-512, 2004.

[38] M. Tsalas, A. Herrmann, A. Kallenbach, H.W. Müller, J. Neuhauser, V. Rhode, N. Tsois, M. Wischmeier, and the ASDEX Upgrade Team. Divertor plasma flow near the lower X-point in ASDEX Upgrade. Plasma Phys. Control. Fusion, 49:857-872, 2007.

[39] L. Aho-Mantila, M. Wischmeier, H.W. Müller, S. Potzel, D.P. Coster, X. Bonnin, G.D. Conway, and the ASDEX Upgrade Team. Outer divertor of ASDEX Upgrade in low density L-mode discharges in forward and reversed magnetic field: I. Comparison between measured plasma conditions and SOLPS5.0 code simulations. Nucl. Fusion, 52:103006, 2012.

[40] A.W. Leonard, G.D. Porter, and R.D. Wood. Radiative divertor plasmas with convection in DIII-D. Phys. Plasmas, 5(5):1736-1743, 1998.

[41] S.I. Krasheninnikov. Physical mechanisms in divertors and their impact on the core. Czech. J. Phys., 48(S2):97-112, 1998.

[42] A. Scarabosio, G. Haas, H. W. Müller, R. Pugno, M. Wischmeier, and ASDEX Upgrade Team. Measurements of neutral gas fluxes under different plasma and divertor regimes in ASDEX Upgrade. J. Nucl. Mater., 390-391:494-497, 2009. 
[43] M. Wischmeier, S. Potzel, M. Bernert, L. Aho-Mantila, H. W. Müller, D. Coster, F. Reimold, and ASDEX Upgrade Team. Symmetries and asymmetries in the divertor detachment in ASDEX Upgrade. 2012 Proc. 24th Int. Conf. on Fusion Energy (San Diego, 2012), EX/P5-34. 